UDO TIETZ

\title{
Gemeinsinn, Gemeinwohl und die Grenzen des „Wir“*
}

Seit dem Ausgang des 18. Jahrhunderts kreist der philosophische Diskurs der Moderne unter immer neuen Titeln um ein einziges Thema: „das Erlahmen der sozialen Bindekräfte, Privatisierung und Entzweiung, kurz: jene Deformation einer einseitig rationalisierten Alltagspraxis, die das Bedürfnis nach einem Äquivalent für die vereinigende Macht der Religion hervorrufen".

Eines dieser Äquivalente ist die Gemeinschaft, mit der Orientierungen zum Zuge kommen sollen, die als Gegeninstanzen gegenüber einer transzendental obdachlosen Moderne aufgeboten werden können: der Gemeinsinn und das Gemeinwohl. Mit der Absicht sie zu „verjüngen“, beschwor Hölderlin sie im Hyperion als Antithese zur beginnenden Moderne in Form eines idealen Griechenlands, ${ }^{2}$ Novalis verklärte sie im Mythos der sinnerfüllten Zeiten, „wo Europa ein christliches Land" und durch „ein großes gemeinschaftliches Interesse" vereint war, ${ }^{3}$ Tönnies setzte sie der starrmechanischen bürgerlichen Gesellschaft entgegen ${ }^{4}$ und in unseren Tagen argumentieren Hermeneutiker und Kommunitaristen für die These, daß ,eine bloß auf die Garantie individueller [...] Grundrechte fixierte Gesellschaft kein Äquivalent für die ,vereinigende Macht der Religion" hervorbringen kann“ weil hierfür eine „ebenso verpflichtende wie identitätsstiftende gemeinsame Konzeption des Guten“ vonnöten sei, eine Konzeption, die ganz wesentlich das ausmacht, was man den kollektiv verbindlichen und kollektiv verbindenden Wert- und Verständnishorizont einer Gemeinschaft nennt, und die allen individuellen Rechten normativ vorgeordnet ist und damit auch deren Grenzen und deren legitimen Anspruch zu bestimmen vermag ${ }^{5}$ - eine Position, die einem differenzblinden Universalismus entgegengesetzt ist und von ihren Vertretern

* Für kritische Hinweise danke ich Cathleen Kantner, Marcus Llanque, Marianne Schark, Herbert Schnädelbach und Tom Seidel.

Habermas 1985b, S. 166.

2 Hölderlin 1970, S. 99 f.

Novalis 1978, S. 507.

4 Tönnies 1991, S. 3 f.

Wellmer 1993, S. 175; Tietz 1995b, S. 208 f. 
selbst als ethnozentristisch, kontextualistisch, partikularistisch oder sogar als relativistisch bezeichnet wird.

Gegen derartige Rekurse auf die Gemeinschaft sind von Seiten der universalistischen Gegenpartei immer wieder Einwände erhoben worden. Zweifel regen sich nicht nur daran, ob sich unter den Bedingungen der Moderne solche Gemeinschaften überhaupt noch ausfindig machen lassen oder ob nicht die Gemeinschaft bloß zu jenen Ideologemen gehört, „auf welche die ,Moderne“ immer wieder zurückgreift, um den Krisen, die ihre Geschichte skandieren und aus denen sich erst das ergibt, was wir ,Moderne' nennen, zu begegnen". ${ }^{6}$ Zweifel regen sich auch daran, ob der Rekurs auf den kollektiv verbindlichen und kollektiv verbindenden Wert- und Verständnishorizont einer partikularen Gemeinschaft möglich und ob er nötig ist, um in modernen Gesellschaften das „Erlahmen der sozialen Bindekräfte, Privatisierung und Entzweiung“, die auf das Konto einer einseitigen rationalisierten Alltagspraxis gehen, kompensieren zu können.

Einwände gegen eine solche Orientierung am Gemeinsinn, dem Gemeinwohl und der Gemeinschaft können auf eine zweifache Weise vorgetragen werden: Als praktische Kritik können sie bestreiten, daß eine solche Orientierung wünschenswert ist, und als theoretische Kritik können sie bestreiten, daß sie sinnvoll ist. Im Folgenden möchte ich zeigen, wie eine solche Orientierung möglich und wie sie nicht möglich ist, wobei ich für die These argumentieren werde, daß sich das, was man landläufig als „Gemeinwohl" bezeichnet (lat., das bonum commune), als ein Wert im Sinne einer an Aristoteles anschließenden hermeneutischen Güterethik verstehen läßt, wie sie in unterschiedlichen Varianten von Hans-Georg Gadamer, Charles Taylor oder Alasdair MacIntyre vertreten wird. Dabei halte ich den Bezug auf gemeinsam geteilte Werte für das normative Selbstverständnis von partikularen Wir-Gemeinschaften für konstitutiv und gehe davon aus, daß das Problem der Werte aus seiner ontologischen Umklammerung herausgelöst und in eine Sprache wertender Unterscheidungen übersetzt werden muß, da sich unter den Bedingungen eines nachidealistischen Denkens solche Werte nicht mehr ontologisch verstehen lassen.

Ich werde für eine hermeneutische und prozeduralistische Auffassung des Gemeinsinns und des Gemeinwohls argumentieren, mit der sich die Grenzen von partikularen Gemeinschaften über gemeinsam geteilte Werte und damit über gemeinsam geteilte Überzeugungen bestimmen lassen, die für die Wertungsoperationen konstitutiv sind, mit denen sich Sprecher auf Dinge und Ereignisse beziehen. Hermeneutisch muß diese Auffassung mindestens aus zwei Gründen sein: erstens, weil sich ein reflektierter Partikularismus, so er sich denn überhaupt noch vertreten läßt, nur noch hermeneutisch vertreten läßt, und zweitens, weil der Zugang zur Problematik des ethischen Selbstverständnisses einer partikularen Gemeinschaft, das sich ohne Bezug auf Werte nicht erklären läßt, nur aus der Perspektive von Teilnehmern erfolgreich sein kann. Und prozedural muß dieser Ansatz sein, weil sich zeigen läßt, daß das Problem der Güter oder Werte, mit denen innerhalb partikularer Wir-Gemeinschaften ja nicht nur partikularistische, sondern mitunter auch universalistische Orientierungen zum Zuge kommen, also Orientierungen, die den Kontext der je eigenen Wir-Gruppe überschreiten, auf einer güterethischen Grundlage nicht zu klären ist.

6 Raulet 1993, S. 77. 
Ein solches Konzept muß zwei Intuitionen Rechnung tragen können: erstens, der aristotelischen Intuition, daß sich "starke Wertungen“, ohne die partikulare Wir-Gruppen nicht zu haben sind, nicht vom Kontext einer partikularen Lebensform trennen lassen, eine Intuition, die ohne Rückgriff auf metaphysische Prämissen vor allem im Umfeld von hermeneutischen und kommunitaristischen Positionen vertreten wird. Und zweitens der kantischen Intuition, daß sich universalistische Prinzipien nicht mit Rekurs auf das Faktizitätsapriori einer partikularen Wir-Gemeinschaft begründen lassen, eine Intuition, die vor allem im Umfeld von diskurstheoretischen Positionen vertreten wird. Darum bezeichne ich die hier entwickelte Position als prozeduralen Aristotelismus.

\section{Gemeinsinn und Gemeinwohl}

Gemeinsinn und Gemeinwohl sind begriffliche Verwandte. Insbesondere wenn vom Zusammenleben der Menschen in kleineren Gruppen die Rede ist, spricht man häufig in einem Atemzug von „Gemeinsinn und Gemeinwohl“, so als ob die beiden Worte dasselbe bedeuten würden. Und dies ist keineswegs ganz unzutreffend, insofern nicht nur der Begriff des „Gemeinwohls“, sondern auch der Begriff des „Gemeinsinns“ eine Bedeutungsdimension anspricht, die sich auf etwas Überindividuelles bezieht, auf etwas, das nicht diesem oder jenem zur Verfügung steht. Schon unsere umgangssprachliche Verwendung dieser Begriffe macht deutlich, daß es sich hierbei um einen „Sinn" handelt, der nicht privatistisch verstanden werden kann. Für den Begriff des Gemeinsinns ist der Bezug auf etwas Überindividuelles konstitutiv, wobei zunächst jedoch unklar ist, was genau unter diesem Überindividuellen zu verstehen ist. ${ }^{7}$ Klar ist jedoch eines: Ein Gemeinsinn ist ein gemeinsam geteilter Sinn. Doch wer oder was teilt hier auf welche Weise welchen Sinn? Die Antwort auf diese Frage ist nicht trivial. Ich denke, daß es auf diese Frage zwei unterschiedliche Antworten geben kann:

Die erste Antwort lautet: Die Gemeinschaft teilt einen sprachlichen Sinn. In diesem Sinne hat bereits Kant den sensus communis als eine Sinnesart bestimmt, die eine ästhetische und eine hermeneutische Dimension aufweist, wobei er letztere als eine ,notwendige Bedingung der allgemeinen Mitteilbarkeit unserer Erkenntnis" ansah, weshalb sie faktisch ,in jeder Logik und jedem Prinzip der Erkenntnisse“ vorausgesetzt sei. ${ }^{8}$ Der Gemeinsinn wird in seiner hermeneutischen Dimension von Kant als ein Mitteilungssinn verstanden, den wir nach Schleiermacher und Dilthey als „Verstehen" bezeichnen. Die Gemeinschaft teilt mit dem Gemeinsinn also einen sprachlichen Sinn dergestalt, daß die Bedeutungen sprachlicher Ausdrücke geteilt, sprich: wechselseitig verstanden werden. Die Gemeinschaft wäre danach eine Sprachgemeinschaft, wie sie in unterschiedlicher Weise von Wittgenstein, Gadamer und Habermas entworfen wurde. Dies wäre die erste Antwort auf die Frage, was für einen Sinn die Gemeinschaft - in Form ihrer Mitglieder - teilt. Die zweite Antwort hingegen lautet: Die angesprochene Ge-

\footnotetext{
7 Vgl. Wingert 1993, S. 12.

8 Der vermögenstheoretisch gedachte Gemeinsinn ist bei Kant ein allgemeines Prinzip, an das sich bei ihm die Hoffnung auf eine allgemeine „Einhelligkeit der Sinnesart" knüpft. Kant 1976, §22.
} 
meinschaft teilt einen Sinn für das Gemeinwohl, wobei ein solcher Sinn im Sinne eines kollektiv verbindlichen und verbindenden Werthorizonts geteilt wird.

Obgleich der gemeinsam geteilte sprachliche Sinn nicht mit dem gleichgesetzt werden darf, was wir hier den Gemeinsinn einer partikularen Wir-Gemeinschaft nennen, so gibt es doch zwischen beiden einen notwendigen Zusammenhang. Der Gemeinsinn als eine Gemeinwohlorientierung wird vom Gemeinsinn, sofern er sprachlich geteilte Bedeutungen impliziert, getragen. Oder anders gefaßt, ein gemeinsames Bedeutungsverstehen muß immer schon garantiert sein, damit es überhaupt eine Orientierung auf das Gemeinwohl geben kann - eine Einsicht, die sich schon bei Max Weber finden läßt, der davon spricht, daß „,das gegenseitige ,Verstehen “ " bzw. die „sinnhafte, Verständlichkeit" des Tuns des Anderen die elementarste Voraussetzung der Vergemeinschaftung ist". ${ }^{9}$ Nur Wesen, die Mitglieder einer intersubjektiven Sprachgemeinschaft sind, sind Wesen, denen wir eine Orientierung auf das Gemeinwohl zumuten und von denen wir eine solche Orientierung auch legitimerweise erwarten können.

"Gemeinsinn“ und „Sprachgemeinschaft" sind demnach nicht deckungsgleiche Begriffe. Denn ansonsten müßten alle Mitglieder einer Sprachgemeinschaft auch einen Gemeinsinn im Sinne einer Orientierung auf das Gemeinwohl teilen. Sie müßten also bereits mit und durch eine gemeinsam geteilte Sprache über einen kollektiv verbindlichen und verbindenden Werthorizont verfügen - was sich jedoch empirisch bestreiten läßt. Der Gemeinsinn im Sinne von sprachlich geteilten Bedeutungen ist lediglich eine notwendige Bedingung einer jeden Orientierung auf ein Gemeinwohl. ${ }^{10}$ Um sinnvoll von einem Gemeinsinn sprechen zu können, müssen die Wesen, denen wir eine Gemeinwohlorientierung zumuten und von denen wir eine solche Orientierung auch erwarten können, nicht Mitglied einer Sprachgemeinschaft sein, sondern lediglich Mitglied einer Sprachgemeinschaft. Es reicht aus, daß sich die Mitglieder einer solchen Gemeinschaft wechselseitig verstehen können. Sie müssen aber nicht unbedingt dieselbe Sprache sprechen, wenngleich dies in vielen Fällen so sein wird. In der Schweiz spricht man bekanntlich mehrere Sprachen und doch erscheint es prima facie nicht absurd, den Schweizern einen Gemeinsinn zuzusprechen.

Dies scheint zunächst zu bedeuten, daß gemeinsam geteilte Überzeugungen bezüglich der Orientierung auf das, was wir das Gemeinwohl nennen, mindestens auf eine zweifache Weise von Relevanz sind: Sie sind erstens für das wechselseitige Verstehen der Mitglieder von Gemeinschaften relevant - ein Verstehen, das hinsichtlich seiner Möglichkeitsbedingungen traditionell im Rahmen der philosophischen Hermeneutik thematisch wird. Wittgenstein, Gadamer, Habermas und Davidson haben auf je unterschiedliche Weise gezeigt, daß ein jedes Verstehen auf ein hermeneutisches Vorverständnis in Form eines "Hintergrunds massiver Übereinstimmungen“ angewiesen ist, der als ein Hintergrund von „gemeinsam geteilten Überzeugungen" zu verstehen ist, ohne den es nicht nur kein Verstehen geben würde, sondern auch keine Wir-Gruppe und

9 Vgl. Weber 1972a, S. 238, Weber 1972b, S. 93.

10 „Die Orientierung an den Regeln der gemeinsamen Sprache ist primär also nur Mittel der Verständigung, nicht Sinngehalt von sozialen Beziehungen" - sie ist noch kein Gemeinsinn im Sinne einer Orientierung auf das Gemeinwohl. Weber 1972a, S. 23. 
damit auch keinen Gemeinsinn und kein Gemeinwohl - egal ob diese Gruppe partikular oder universell verstanden wird.

Gemeinsam geteilte Überzeugungen sind aber nicht nur für ein wechselseitiges Verstehen relevant, sondern auch für die Konstitution von Wir-Gemeinschaften in einem engeren Sinn, also auch für den Gemeinsinn und das Gemeinwohl einer partikularen Wir-Gemeinschaft. Wir müssen daher zunächst die Frage klären, welche Rolle genau diese Überzeugungen bei der Konstitution von partikularen Wir-Gruppen spielen, eine Frage, die beantwortet sein muß, damit wir die Frage nach dem Gemeinsinn im Sinne der Orientierung auf ein Gemeinwohl überhaupt stellen können. Ich frage zunächst also nicht nach dem Gemeinsinn und dem Gemeinwohl, um dann auf die Wir-Gruppe zu sprechen zu kommen, sondern umgekehrt nach der Rolle von gemeinsam geteilten Überzeugungen bei der Konstitution von Wir-Gemeinschaften.

Der Grund für dieses Vorgehen ist, daß die Begriffe „Gemeinwohl" und „Gemeinsinn" ohne jenen der "Gemeinschaft" überhaupt nicht zu verstehen sind - wobei der Begriff der "Gemeinschaft" der grundlegendere Begriff ist, da dieser ohne jenen verstanden werden kann, nicht aber jener ohne diesen. Und der Begriff des „Gemeinwohls“ verweist ja auch schon in seiner Bedeutung auf den Begriff der "Gemeinschaft", insofern ein Gemeinwohl ja nichts anders ist, als das Wohl einer Gemeinschaft - was dann analog auch für den Begriff des „Gemeinsinns" gilt, insofern wir darunter eine Orientierung am Gemeinwohl und damit an der Gemeinschaft verstehen.

Wer also über das Gemeinwohl und den Gemeinsinn redet, der darf nicht von der Gemeinschaft schweigen wollen. Denn immer wenn man vom Gemeinwohl spricht, spricht man automatisch von einer Gemeinschaft, um deren Wohl es geht. Darum ist zunächst zu klären, was genau unter einer solchen Gemeinschaft zu verstehen ist. Wir müssen anscheinend zunächst die Frage nach der Rolle von gemeinsam geteilten Überzeugungen bei der Konstitution von partikularen Wir-Gruppen beantworten, die kleiner sind als die gesamte Menschheit - also jener Wesen, die sagen können: „Wir sind vernünftig“. Diese Frage könnte man auch so formulieren: Es gibt Gemeinschaften - wie sind sie möglich?

Die Antworten auf diese Frage sind notorisch unklar. Lediglich, daß Überzeugungen hierbei eine Rolle spielen, scheint nicht kontrovers zu sein. Zwar hat sich in der Philosophie und in den Sozialwissenschaften inzwischen die Auffassung durchgesetzt, daß die Gemeinsamkeit von lebensweltlich situierten Wir-Gruppen, die sich nicht strategisch integrieren, als eine Gemeinsamkeit verstanden werden muß, die auf einem normativen Konsens beruht. In der Phänomenologie von Husserl, der philosophischen Hermeneutik von Gadamer, der philosophischen Anthropologie von Gehlen, der Konzeption der Sprachspiele von Wittgenstein und der Diskurstheorie von Apel und Habermas finden sich diesbezüglich ähnliche Intuitionen wie in der Soziologie bei Emile Durkheim, Ferdinand Tönnies, George Herbert Mead und Max Weber. Und auch die postempiristische Wissenschaftstheorie kam mit Thomas S. Kuhn zu einem ähnlichen Befund. Denn auch Kuhn meinte ja, daß die Begriffe des „Paradigmas“ und der „,Gemeinschaft" in einem zirkulären Explikationsverhältnis stünden. Wie aber dieser Konsens genau verstanden werden muß und wie sich die Konsense von unterschiedlichen Gemeinschaften zueinander verhalten, darüber gehen die Meinungen auseinander. 
Wie immer man sich nun in dieser Debatte verortet, ob man eher der partikularistischen oder der universalistischen Position zuneigt, zweierlei wird sich kaum bestreiten lassen: Erstens, daß solche Konsense für partikulare Wir-Gruppen konstitutiv sind. Und zweitens, daß sie die Einheit des Kollektivs verbürgen, das den Bezugspunkt für die Gemeinsamkeit aller Angehörigen bildet, die darin zum Ausdruck kommt, daß diese von sich in der ersten Person Plural sprechen können. Die Mitglieder solcher Kollektive sagen „wir" - im Gegensatz zu ,ihnen“, womit in der Regel jene gemeint sind, die nicht zur je eigenen Wir-Gruppe gehören. Aus diesem Tatbestand hat Richard Rorty die Schlußfolgerung gezogen, daß wer „wir" sagt, damit auf einen Kontrast zu „ihnen“ abhebt, „die ebenfalls Menschen sind - aber Menschen von der falschen Sorte", "wobei er diese These mit der ethnozentristischen These in Verbindung gebracht hat, daß die Unterscheidung zwischen ,uns“ und, ihnen“ identisch ist mit der Einteilung des Menschengeschlechts, in diejenigen, vor denen man seine Überzeugungen rechtfertigen muß, und die übrigen. Die erste Gruppe - der ethnos - umfaßt diejenigen, mit deren Meinungen man genügend übereinstimmt, um ein fruchtbares Gespräch möglich zu machen", und die zweite Gruppe umfaßt jene, bei denen von einer derartigen Übereinstimmung nicht gesprochen werden kann. Danach ist dann das, was als „rational oder fanatisch gilt", nicht mehr absolut, sondern ,relativ und richtet sich nach der Gruppe, vor der man sich rechtfertigen zu müssen meint; es richtet sich nach dem Korpus gemeinsamer Überzeugungen, das den Bezug des Wortes, wir ${ }^{6}$ bestimmt ${ }^{\star 1}{ }^{12}$

Die Pointe des Ethnozentrismus besteht danach in einer Relativierung des Rationalitätsbegriffs durch eine partikularistische Begrenzung des „logischen Raums des Begründens " und in der damit zusammenhängenden Einteilung des Menschengeschlechts in zwei Gruppen: in die, ,vor denen man seine Überzeugungen rechtfertigen muß, und die übrigen"! So verstanden, wären partikularistische Wir-Gemeinschaften Rechtfertigungsgemeinschaften, insofern die Rechtfertigungsbedürftigkeit und die Rechtfertigungsfähigkeit von Überzeugungen nur in den von innen gezogenen Grenzen von partikularen Wir-Gemeinschaften einen Sinn haben soll. ${ }^{13}$

Ich halte diese Begründung nicht für plausibel. Zwar können es sich Partikularisten als Verdienst anrechnen, die Frage nach den Grenzen des Wir gestellt zu haben - etwa im Gegensatz zu Universalisten, die mehr damit beschäftigt sind, die Herausforderungen des Kontextualismus abzuwehren. Die hierauf gegebene Antwort vermag jedoch nicht zu überzeugen - zumindest nicht in dieser Form. Denn sie kann mit einem Einwand konfrontiert werden, der sich durch den Partikularisten nicht zurückweisen läßt, da er sich auf eine Prämisse stützt, die er selbst als gültig akzeptiert, auf die Prämisse nämlich, daß ,die Überzeugungen der Menschen nicht in einem freischwebenden Verhältnis zur nichtmenschlichen Umwelt stehen können und daß die meisten unserer (und überhaupt jedermanns) Überzeugungen wahr sein müssen“. ${ }^{14}$

\footnotetext{
$"$ Rorty 1989, S. 307.

12 Rorty 1988, S. 27 und S. 85.

13 Vgl. Tietz 2001, S. 89 f.

14 Rorty 1994, S. 23 und S. 93; Vgl. Davidson 1982, S. 327; Davidson 1991, S. 1007; Vgl. Tietz 2002 b, Kap. 3.
} 
Ohne hier weiter auf Rortys Argumentation einzugehen, sei zumindest festgestellt, $\mathrm{da} ß$ gegen diese Variante des epistemischen Partikularismus mindestens drei Argumente sprechen, die gleichzeitig für einen hermeneutischen Universalismus sprechen: Erstens, daß die ethnozentrische Begrenzung des Raums der Gründe mit dem „holistischen Charakter der Überzeugungszuschreibung" unvereinbar ist; zweitens, daß sich gar nicht angeben läßt, was genau unter dem „Wir" zu verstehen ist; und drittens, daß sich unter der Voraussetzung eines uneingeschränkten Holismus der Begriff der WirGruppe auflöst, wodurch wir gerade das verlieren würden, was wir suchen: Gemeinschaften, die kleiner als die gesamte Menschheit sind. Damit würden wir natürlich auch das Gemeinwohl und den Gemeinsinn verlieren, weil wir nicht angeben könnten, um wessen Wohl es eigentlich bei dem besagten Gemeinwohl geht. ${ }^{15}$

Die von Rorty unterstellte Rationalität ist eine kontrafaktische Präsupposition, die sich nicht partikularistisch verstehen läßt, sondern universalistisch verstanden werden muß. „Alles, womit wir Rationalitätsansprüche verbinden, steht schon unter dieser Präsupposition. Sie hat die Form einer Behauptung der ersten Person Singular oder Plural ,Ich bin vernünftig/Wir sind vernünftig، "l6 Und dieses „Wir" fällt nicht mit dem „Wir" der je eigenen Wir-Gemeinschaft zusammen, sondern mit jenem ,Wir", dessen Verhalten sich auf der Basis unseres Interpretationsschemas zumindest als ,,minimal vernünftig" bezeichnen läßt. ${ }^{17}$

\section{Verstehen und Rechtfertigen}

Offenkundig muß man unterscheiden, zwischen einem Gebrauch von „Wir“ im Sinne einer Rationalitätsunterstellung und einem Gebrauch von ,Wir", der sich auf eine spezifische Gruppe bezieht und zur Abgrenzung von anderen Individuen und Wir-Gruppen dient. Beide Fälle sind nicht identisch: Im ersten Fall handelt es sich um eine Verständlichkeitsunterstellung, im zweiten hingegen um eine Identifikation auf der Basis dieser Präsupposition. Mit der Verständlichkeitsunterstellung konstituieren die Mitglieder eine prinzipiell offene und unbegrenzte Kommunikationsgemeinschaft, während sie mit der Identifikation daraus jene ausgrenzen, die zu einem in Raum und Zeit lokalisierbaren Kollektiv gehören.

Ich schlage daher vor, daß Wort „Wir" mit Indizes zu versehen. Im ersten Fall grenzen sich die Sprecher von Nicht-Sprechern ab $\left(W_{i r}\right)$, im zweiten Fall grenzen sich bestimmte Sprecher gegenüber einer Reihe von anderen Sprechern ab, so daß sie eine partikulare Wir-Gemeinschaft im Verhältnis zu anderen Wir-Gemeinschaften bilden $\left(\mathrm{Wir}_{2}\right)$. Der erste Fall bezieht sich umstandslos auf all jene Wesen, die Kant als vernunftbegabte Wesen bezeichnet hatte, weshalb auf dieses Wir ${ }_{1}$ auch das Prädikat „rational ${ }_{1}$ " im Sinne von nicht nichtvernünftig Anwendung findet. Auch der zweite Fall bezieht sich auf vernunftbegabte Wesen, aber in einer anderen Weise und in einem eingeschränkten Sinn. Beide Fälle sind nicht identisch. Dennoch stehen sie in einem

\footnotetext{
15 Vgl. Tietz 2001, S. 90 f.

16 Schnädelbach 1992a, S. 72.

17 Vgl. Rorty 1988, S. 18; Putnam 1982, S. 162.
} 
wechselseitigen Explikationsverhältnis. Denn nur Wesen, die Mitglied der ersten Wir - $^{-}$ Gemeinschaft sind, denen wir also Vernunft und Rationalität zusprechen, sind Wesen, die auch in einem engeren Sinn zu ,uns" gehören können, weshalb auf dieses $\mathrm{Wir}_{2}$ das Prädikat ,rational ${ }_{2}$ " Anwendung findet, das sich nun aber auf die partikulare Rationalität eines Kollektivs bezieht, das kleiner ist als die gesamte Menschheit. Von daher ist $\mathrm{Wir}_{1}$ stets größer als $\mathrm{Wir}_{2}$. Man muß ein Mitglied der Wir ${ }_{1}$-Gemeinschaft sein, damit man ein Mitglied von $\mathrm{Wir}_{2}$-Gemeinschaften sein kann. Diese Feststellung scheint mir nicht trivial. Denn wenn dies richtig ist, wenn also nur Wesen zu „uns" gehören können, die Mitglied einer offenen und unbegrenzten Wir $_{1}$-Gemeinschaft sind, dann bedeutet dies, daß ein richtig verstandener Partikularismus den Universalismus zu seiner Voraussetzung hat! Ein recht verstandener Partikularismus ist ohne einen recht verstandenen Universalismus nicht zu haben!

Dies ist auch ein Grund dafür, daß es auf die Frage wer oder was wir sind, so verschiedene Antworten geben kann. ,Jede bestimmt eine andere Art des ,Wir'-Sagens; und jede Art des ,Wir'-Sagens bestimmt eine andere Gemeinschaft, so daß wir uns in einer Vielzahl von Gemeinschaften wiederfinden. Das legt den Gedanken nahe, daß wir uns selbst mehr oder weniger für diejenigen halten, die ,wir ${ }^{6}$ sagen, und verweist auf eine große Gemeinschaft, der die Mitglieder aller besonderen Gemeinschaften angehören - die Gemeinschaft derer, die über und zu jemandem ,wir' sagen, ganz gleichgültig, ob nun die Mitglieder dieser besonderen Gemeinschaften einander kennen oder nicht. "

Ein Gemeinsinn im Sinne einer Orientierung auf das Gemeinwohl einer partikularen $\mathrm{Wir}_{2}$-Gemeinschaft ist also ohne einen Gemeinsinn im Sinne von sprachlich geteilten Bedeutungen nicht zu haben. Nur vernunftbegabte Wesen, die kommunizieren und sich verstehen, können Mitglieder von $\mathrm{Wir}_{2}$-Gemeinschaften sein. Dieses Resultat ist allerdings noch unbefriedigend, zumal es eher auf eine Bestätigung von kantischen als von aristotelischen Intuitionen hinauszulaufen scheint. Gesucht war aber eine Position, die ich als einen reflektierten Partikularismus bezeichnet habe, eine Position, die es erlaubt, mit Rekurs auf gemeinsam geteilte Überzeugungen die Grenzen von $\mathrm{Wir}_{2}$ Gemeinschaften $\mathrm{zu}$ bestimmen - wobei ein solcher Partikularismus eine Bedingung erfüllen sollte, die Bedingung nämlich, mit dem hermeneutischen Universalismus kompatibel zu sein. Ein reflektierter Partikularismus muß ein Partikularismus sein, der sich mindestens in hermeneutischer Hinsicht widerspruchsfrei mit dem Universalismus verträgt. Wie bereits bemerkt: Wenn sich ein reflektierter Partikularismus überhaupt vertreten läßt, muß er sich in einer hermeneutisch reflektierten Variante vertreten lassen, d. h. in einer Weise, die die Partikularisierung des logischen Raums der Gründe ausschließt!

Dies scheint mir bei Rorty, Taylor und MacIntyre aber nicht der Fall zu sein - was Rorty inzwischen selbst auch zugegeben hat. Denn Rorty meint heute, daß „der holistische Charakter der Zuschreibung intentionaler Zustände [...] die Zersplitterung des logischen Raums, in dem Gründe genannt und verlangt werden“, verbietet. „Es gibt tatsächlich nur einen einzigen logischen Raum. " ${ }^{\text {19 }}$ Dennoch ist das vorläufige Ergebnis nicht nur negativ. Zum einen haben wir mit diesem Ergebnis notwendige Bedingungen

${ }_{18}$ Brandom 2000, S. 36.

19 Rorty 2001 , S. 93 f. 
einer jeden Gemeinschaftsbildung benannt. Und zum anderen wurde deutlich, wie sich ein vernünftiger Begriff einer partikularen $\mathrm{Wir}_{2}$-Gemeinschaft und damit auch ein sinnvoller Begriff des Gemeinwohls und des Gemeinsinns nicht gewinnen läßt. Es zeigt also, wie es nicht geht. Es gibt uns damit aber auch einen Fingerzeig, wie sich ein partikularistischer Gemeinschaftsbegriff gewinnen läßt.

Ein solcher Begriff läßt sich gewinnen, wenn wir zunächst die These aufgeben, der „logische Raum des Begründens“ lasse sich partikularistisch begrenzen; wenn wir zweitens den uneingeschränkten Holismus so einschränken, daß die Konsequenz vermieden wird, die den Begriff der Sprach- und damit der $\mathrm{Wir}_{2}$-Gemeinschaft auflöst, wenn wir drittens die Frage nach der Rechtfertigung von Überzeugungen dergestalt liberalisieren, daß sie nicht mehr als eine Frage des Alles-oder-Nichts erscheint, sondern als eine Frage des Mehr-oder-Weniger; und wenn wir viertens die Grenzen des Wir nicht mehr mit Rekurs auf den „logischen Raum der Begründens“ zu bestimmen versuchen, sondern über einen kollektiv verbindlichen und kollektiv verbindenden Werthorizont einer partikularen $\mathrm{Wir}_{2}$-Gemeinschaft.

\section{Sprache, Werte und die Identität der Gemeinschaft}

Wer die Grenzen des Wir mit Rekurs auf einen kollektiv verbindlichen und kollektiv verbindenden Werthorizont bestimmen will, der muß etwas über wertrational integrierte soziale Beziehungen sagen können. Es erscheint daher nötig, sich die Verbindungsart anzusehen, die für die Gemeinschaftsbildung von partikularen $\mathrm{Wir}_{2}$-Gruppen konstitutiv ist. Dabei können wir von einer Feststellung ausgehen, die sich bereits in der Kritik der reinen Vernunft findet. Hier stellt Kant fest, daß ,das Wort Gemeinschaft [...] in unserer Sprache zweideutig“ ist: es „kann soviel als communio, aber auch als commercium bedeuten“, ${ }^{20}$ wobei Kant unter einer Gemeinschaft im Sinne eines „commercium" einen Verkehrszusammenhang versteht, in dem eine Vielheit gleichrangiger Komponenten „in durchgängiger Gemeinschaft der Wechselwirkung untereinander [...] stehen “21 ohne dabei ein Gemeinsames zu bewirken, wohingegen er unter einer Gemeinschaft im Sinne einer „communio“ einen Verkehrszusammenhang versteht, in dem die Vielheit der Komponenten dadurch integriert sind, daß sie etwas miteinander machen und sich so miteinander vereinen - wobei hier die Komponenten nicht notwendigerweise gleichrangig sein müssen.

So sah es auch Ferdinand Tönnies, der die Gesellschaft als „ein [...] Nebeneinander voneinander unabhängiger Personen" versteht, die nichts miteinander machen, die allenfalls etwas vereinbaren, sich aber dabei nicht vereinen, der er die Gemeinschaft gegenüberstellt, die er als ein Zusammenwirken der Menschen bestimmt, das „im Besitz und Genuß gemeinsamer Güter" gründet. ${ }^{22}$ Im Unterschied also zu gewaltsam konstituierten Sozialverbänden spricht Tönnies mit den Begriffen „Gemeinschaft“ und „Gesellschaft" zwei elementare Grundformen des sozialen Lebens an, mit denen er die Ver-

20 Kant 1979, A 213.

21 Ebd.

22 Tönnies 1991, S. 4 u. 20. 
bundenheit im Sozialen zu erklären sucht, so diese sich auf „Verhältnisse gegenseitiger Bejahung" beziehen lassen, auf Verhältnisse also, die sich nicht auf bloße Macht und Gewalt gründen.

Zwar dürfen wir nicht vergessen, daß der Gemeinschaftsbegriff bei Tönnies immer auch als ein lebensphilosophischer Kontrastbegriff zur starren und mechanischen Gesellschaft fungiert. Die Gemeinschaft als Gebietskategorie des Vorkapitalistischen wird so zur Verherrlichung von vermeintlich „organischen Zuständen“ und zugleich zur Kampfparole gegen die mechanisierenden und damit kulturzerstörenden Wirkungen des kapitalistischen Systems. Insofern handelt es sich bei Tönnies immer auch um eine „Apologie der Gemeinschaft gegen die vergesellschaftenden Tendenzen von Staat und Wirtschaft". ${ }^{23}$ Dies ist aber eben nur eine Dimension dieses Begriffs. Die andere Dimension ist die, daß Tönnies mit diesem Begriff auf einen Verkehrszusammenhang abhebt, in dem die Gemeinschaftsmitglieder nicht systemisch, sondern über den kollektiv verbindlichen und kollektiv verbindenden Werthorizont einer partikularen $\mathrm{Wir}_{2}$ Gemeinschaft integriert sind.

Der Begriff der Gemeinschaft läßt sich bei Tönnies also in einer zweifachen Weise verstehen: Er läßt sich erstens als eine lebensphilosophische Antithese und damit als „eine Art Gegenideologie der industriellen Gesellschaft" verstehen. ${ }^{24}$ In diesem Sinne dürfte er uns heute kaum noch etwas zu sagen haben, weil die Beschwörung jener Zeiten, die einst als die sinnerfüllten galten, nicht mehr zu überzeugen vermag. Und er läßt sich zweitens als ein Begriff verstehen, der sich auf eine Integrationsform bezieht, die über gemeinsam geteilte Werte läuft, die dann das ausmachen, was man den kollektiv verbindlichen und kollektiv verbindenden Werthorizont einer partikularen Wir 2- $^{-}$ Gemeinschaft nennen kann - wobei Tönnies im Gegensatz zu homogenisierenden Gemeinschaftsmodellen, in denen die Einheit in einem starken Sinne als Gleichheit der Mitglieder verstanden wird, die Auffassung vertritt, daß die Einheit erstens prozedural zu verstehen sei, nämlich aus einem „Verhältnis gegenseitiger Bejahung" und daß diese Einheit zweitens nur eine „Einheit des Differenten“ sein könne.

Nur in diesem zweiten Sinne werde ich den Gemeinschaftsbegriff in der Folge weiter verwenden und unter einer partikularen Gemeinschaft eine über gemeinsam geteilte Werte integrierte Gemeinschaft verstehen. Wertegemeinschaften sind normativ integrierte Gemeinschaften, die sich auf das Gemeinwohl im Sinne dessen beziehen, was aufs Ganze gesehen für die Mitglieder dieser Gemeinschaft gut oder besser ist. Dies ist freilich erst die halbe Wahrheit. Denn die Frage, wie diese Integration genau zu verstehen ist, ist damit noch nicht beantwortet. Die Frage nämlich, was Werte sind und wie diese innerhalb einer solchen Gemeinschaft geteilt werden, läßt sich mit Tönnies nicht mehr klären.

Wir stoßen bezüglich der Gemeinschaftsproblematik hier auf ein Problem, das bis dahin weder in der Soziologie noch in der Philosophie eine einigermaßen plausible Lösung gefunden hat. Hier wie dort fehlten die konzeptionellen Mittel, die Art und Weise dieser Integration plausibel zu machen, so daß man meist mit dem Verweis auf große Synthesen operierte. Danach geht eine Gemeinschaft aus der „Synthese“ der Per-

\footnotetext{
23 Plessner 1955, S. 341.

24 Ebd.
} 
sonen hervor, die sie umfaßt. So sahen es Durkheim und Simmel und so sahen es Husserl und Adler - weshalb man in diesem Zusammenhang auch von einem ,SozialApriori" sprach. Am nächsten kam wohl George Herbert Mead der Lösung des Problems, der im Rahmen des symbolischen Interaktionismus ein Konzept der Perspektivenverschränkung entwarf, das den kollektiv verbindlichen und kollektiv verbindenden Werthorizont einer partikularen Wir ${ }_{2}$-Gemeinschaft aus den symbolischen Äußerungen von sprach- und handlungsfähigen Subjekten erklärte.

Diese Intuition läßt sich hermeneutisch dergestalt konkretisieren, daß wir die Integration als eine sprachlich vermittelte und normativ gesteuerte Integration verstehen. ${ }^{25}$ Die Identität partikularer $\mathrm{Wir}_{2}$-Gemeinschaften wäre danach eine Identität, die sprachlich über gemeinsam geteilte Werte vermittelt ist, wobei unter solchen Werten abstrakte Gegenstände zu verstehen sind, auf die wir uns mit substantivierten Prädikaten beziehen. "Schönheit" oder „Fitneß" stellen in diesem Sinne genauso Werte dar, an denen sich eine Gemeinschaft in ethisch-existenzieller Hinsicht orientieren kann, wie "Gerechtigkeit" oder „Solidarität“ ${ }^{26}$ Werte sind Pseudogegenstände und Wertbegriffe ana$\log$ zu den Begriffen, die man mit als Kant „Prädikate möglicher Urteile ${ }^{، 27}$ bezeichnen kann, „Prädikate möglicher Beurteilungen“, wobei wir diese Prädikate als Regeln verstehen sollten, denen wir bei Beurteilungen in der Verwendung der jeweiligen Prädikatausdrücke folgen. Wenn man also das Wertproblem richtig stellt, d. h. in Übereinstimmung mit der Grammatik der evaluativen Rede, dann wären das, was den Werten entspricht, die intersubjektiven Verwendungsregeln von Wertprädikaten, über die wir als Wertende nicht frei verfügen können, weil sie eine subjektunabhängige Geltung haben - womit zumindest angedeutet ist, auf welcher Grundlage eine Auseinandersetzung über Wertfragen überhaupt nur geführt werden kann: nämlich auf einer anerkennungstheoretischen Grundlage. ${ }^{28}$

Damit lassen sich zwei wichtige Teilergebnisse festhalten: Erstens, daß die Identitätsbedingungen auf den Begriff des „Gemeinwohls“ verweisen, insofern diese kollektive $\mathrm{Wir}_{2}$-Identität auf eine nicht-kontingente Weise mit bestimmten Wertungen ${ }^{29}$ und damit mit bestimmten Überzeugungen und Wünschen der Kollektivmitglieder verknüpft ist, die sich auf das beziehen, was aus der Sicht der Kollektivmitglieder aufs Ganze gesehen gut oder besser für die $\mathrm{Wir}_{2}$-Gruppe ist; und zweitens, daß ein solcher Konsens durch die Kanäle der sprachlichen Kommunikation hindurch muß - wobei

${ }^{25}$ Tietz 2002b, Kap. 2.

${ }^{26}$ Vgl. Luhmann 1986, S. 213. Es wäre verfehlt, das Problem des Gemeinschaft auf den Bereich der Politik einzugrenzen, da die Orientierung an gemeinsam geteilten Werten nicht nur für diesen Bereich charakteristisch ist. Das Problem der Gemeinschaft muß zunächst unabhängig von etwaigen politischen Konsequenzen durchdacht werden, weil viele $\mathrm{Wir}_{2}$-Gemeinschaften keine politischen Gemeinschaften sind. Die soziale Welt ist ein Konglomerat sozialer $\mathrm{Wir}_{2}$-Gemeinschaften, die sich in teils konzentrischen, teils sich überlappenden sozialen Kreisen aufeinander beziehen, wobei es die politische Gemeinschaft ist, die nach liberalem Verständnis, in der Außenbeziehung gleichberechtigt mit andern politischen Gemeinschaft agiert und nach innen eine Einheit in der Differenz verwirklichen soll, so daß Raum für eine differierende Vielfalt von Lebensformen geschaffen wird.

${ }^{27}$ Kant 1979, A 69.

${ }^{28}$ Vgl. dazu Schnädelbach 2001; Tietz 2002b, Kap. 2.

29 Vgl. dazu Taylor 1988, S. 36 f. 
solch ein kommunikativ erzieltes Einverständnis immer propositional differenziert ist, was gleichzeitig der Grund dafür ist, daß es sich nicht von außen induzieren läßt. Ein kommunikativ erzieltes Einverständnis muß immer von den Beteiligten als gültig akzeptiert werden, sonst ist es eben kein kommunikativ erzieltes Einverständnis. Und ein solches „Einverständnis beruht auf gemeinsamen Überzeugungen“ ${ }^{30}$

Die Einheit des Kollektivs bildet also den Bezugspunkt für die Gemeinsamkeit aller Angehörigen. Diese Gemeinsamkeit kommt darin zum Ausdruck, daß die Mitglieder des Kollektivs sich mit ihrem Kollektiv identifizieren und von sich in der ersten Person Plural sprechen können. „Unter einer kollektiven oder Wir-Identität verstehen wir das Bild, das eine Gruppe von sich aufbaut und mit dem sich die Mitglieder identifizieren. Kollektive Identität ist eine Frage der Identifikation seitens der beteiligten Individuen. Es gibt sie nicht , an sich", sondern nur in dem Maße, wie sich bestimmte Individuen zu ihr bekennen. Sie ist so stark oder so schwach, wie sie im Denken und Handeln der Gruppenmitglieder lebendig ist und deren Denken und Handeln zu motivieren vermag $^{\text {31 }}$ - wobei der Identitätsbegriff in diesem Zusammenhang für die Fähigkeit der Kollektivmitglieder steht, sich auf der Grundlage eines reflektierten Selbstverhältnisses mit dem Kollektiv zu identifizieren, das sie sind und das sie künftighin sein wollen.

Was ich vorschlage, ist somit eine hermeneutische Deutung der Tiefengrammatik des Problemzusammenhangs Gemeinsinn-Gemeinwohl-Gemeinschaft, da unter den Bedingungen der Moderne die hermeneutische Idee des Gesprächs jeder Gemeinschaftlichkeit als eine notwendige Idee eingeschrieben ist, so dieses Gespräch um die Frage kreist, was aus der Sicht der Mitglieder dieser Gemeinschaft aufs Ganze gesehen gut oder besser für sie ist - und zwar nicht als die Idee eines utopischen Endzustandes, der nur noch jenseits der Geschichte zu denken wäre, sondern als Idee eines hermeneutischen Minimums, ohne dessen Realisierung sich gemeinschaftliche Wertorientierungen nur noch dogmatisch rechtfertigen ließen.

Mit dieser hermeneutischen Deutung lassen sich zwei alte Intuitionen auf neue Weise zusammenführen. Die aristotelische Intuition, daß sich ,starke Wertungen ${ }^{632}$ nicht vom Kontext einer partikularen Lebensform trennen lassen und die hermeneutische Intuition, $\mathrm{da}$ nicht nur die Sprache in dem Gespräch gründet, das wir sind, ${ }^{33}$ sondern auch die Gemeinschaft, insofern diese, wie vermittelt auch immer, aus der Ich-Du-Perspektive von Kommunikationsteilnehmern ihr normatives Selbstverständnis schöpft. Beide, die Sprache und die Gemeinschaft, müssen vom „Gespräch" her gedacht werden - wobei ich mich mit der These, daß die Ich-Du-Beziehung als die ,grundlegende soziale Struktur" betrachtet werden muß, auf die Prämissen des methodischen Individualismus verpflichte. Wenn man den Problemkomplex Gemeinsinn-Gemeinwohl-Gemeinschaft von den bekannten Surrogatbildungen entkoppeln will, dann muß der Begriff einer diskursiven Gemeinschaft, eines Wir, aus den kommunikativen Bestandteilen des Gesprächs aufgebaut werden.

\footnotetext{
${ }^{30}$ Habermas 1985a, Bd. 1, S. 386.

31 Assmann 1992, S. 132.

32 Vgl. Taylor 1988, S. 11 ff.; Taylor 1994a, S. 34 ff; Vgl. Tietz 1996; Tietz 1998.

33 Vgl. Heidegger 1981, S. 38 f.
} 
Dies impliziert eine doppelte Gegnerschaft: Der prozedurale Aristotelismus, der von den Prämissen des methodischen Individualismus ausgeht, ist zunächst dem Atomismus entgegengesetzt, der sich auf den kollektiv verbindlichen und kollektiv verbindenden Wert- und Verständnishorizont einer Gemeinschaft zwar bezieht, diesen aber nicht erklären kann; und er ist dem Kommunitarismus entgegengesetzt, der über die wertrational integrierte Gemeinschaft so redet, als handele es sich hierbei um ein Subjekt im Großformat und der diese Wertungen entweder mit Rekurs auf Traditionen begründet, wie dies bei Ritter, Gadamer und MacIntyre der Fall ist, die an die Aristotelische Tugendethik anschließen, oder mit Rekurs auf eine „umfassendere Ordnung“, „die ihre Ansprüche an uns stellen kann", wie dies etwa von Taylor im Rahmen einer an Aristoteles anschließenden Güterethik versucht wird. ${ }^{34}$ Beide, der Atomismus und der Kommunitarismus, verkennen den diskursiven Charakter der sozialen Struktur und beide verfügen über keine plausible Theorie der Werte, die nach der hier vertretenen Auffassung ebenfalls aus unseren diskursiven Praktiken des Gebens und Nehmens von Gründen rekonstruiert werden muß.

\section{Methodischer Individualismus und das Problem der Gemeinschaft}

Gegen meinen Vorschlag, das Problem der Gemeinschaft und des Gemeinsinns hermeneutisch über gemeinsam geteilte Werte und damit auch über die gemeinsam geteilten Überzeugungen von kompetenten Sprechern einer partikularen Wir ${ }_{2}$-Gemeinschaft anzugehen, können zwei Einwände geltend gemacht werden: Erstens könnte man einwenden, daß ein methodischer Individualismus auf eine Ontologie verpflichtet sei, die mit einem „ungebundenen Selbst" operiere und deshalb den "Sinn gegenseitiger Verpflichtungen“ verfehlen muß, da diese „nur von gebundenen Individuen aufrechterhalten werden (können), die einen starken Gemeinschaftssinn teilen" - weshalb der methodische Individualismus dann auch „,soziale Güter als Verkettungen von individuellen Gütern auffassen “35 müsse; und zweitens könnte man einwenden, daß zu einer recht verstandenen Gemeinschaft nicht nur die hier in Anschlag gebrachte Komponente gemeinsam geteilter Werte und Überzeugungen gehöre, sondern stets auch eine gefühlsmäßige Basis, ohne die jede Gemeinschaft in der Luft hinge - wobei man den ersten Einwand den kommunitaristischen und den zweiten den affektualistischen Einwand nennen könnte.

Für beide Einwände finden sich Argumente. Der affektualistische Einwand könnte sich etwa auf Max Weber berufen, der die Auffassung vertrat, daß eine Gemeinschaftsbildung ohne Zusammengehörigkeitsgefühle nicht zu haben sei. "Vergemeinschaftet" heißt bei ihm eine soziale Beziehung, „wenn und soweit die Einstellung des sozialen Handelns [...] auf subjektiv gefühlter (affektueller oder traditioneller) Zusammengehö-

34 Taylor 1995, S. 102.

35 Taylor 1994b, S. 107 u. 102. 
rigkeit der Beteiligten beruht“ “ ${ }^{36}$ Mit dieser Definition, mit der er terminologisch an Tönnies anschließt, widerspricht Weber zwar nicht per se der Auffassung, daß Gemeinschaften an gemeinsam geteilte Werte gebunden sind, die Vergemeinschaftung auf der Basis einer subjektiv gefühlten Zusammengehörigkeit der Beteiligten scheint für ihn jedoch einen besonderen Status zu besitzen.

Nun geht es mir hier nicht darum, den sozialdiagnostischen Wert einer Soziologie zu bestreiten, die den Begriff des Gefühls ins Zentrum der Analyse von Gemeinschaften stellt, einer Soziologie, die für die Analyse von Partnerschaften, religiösen Sekten und vielleicht auch für die von politischen Parteien durchaus von Wert sein dürfte. Ich bezweifle jedoch, daß eine subjektiv gefühlte Zusammengehörigkeit für jede Form der Gemeinschaftsbildung konstitutiv ist und daß eine Gemeinschaft, die tatsächlich auf solch einer Zusammengehörigkeit beruht, sich auf einen normativen Konsens gründet, der sich auf Fragen des "guten" oder des "gelingenden Lebens" bezieht. ${ }^{37}$

Eine gefühlte Zusammengehörigkeit ohne Bezug auf Fragen des guten Lebens könnte den Verbindlichkeitscharakter, der sich mit dem Guten verbindet - das "Sollen“ -, nicht erklären. Zudem sind Gefühle nicht immer wechselseitig, wie jeder unglücklich Verliebte weiß. Und es soll ja sogar Menschen geben, die emotionale Beziehungen überhaupt nicht eingehen, ohne daß wir deshalb sagen würden, daß sie keine Mitglieder von partikularen $\mathrm{Wir}_{2}$-Gemeinschaften sein können. Die Vergemeinschaftung von sozialen Beziehungen auf der Basis einer affektuellen Zusammengehörigkeit der Beteiligten ist darum ein Sonderfall der Vergemeinschaftung, der von jenen Vergemeinschaftungsformen abhängig bleibt, die Max Weber ,zweck-“ und ,wertrational " nennt. ${ }^{38}$ Und diese Vergemeinschaftung erfolgt über die gemeinsam geteilten Werte, die jene präferieren, die zu der Gemeinschaft gehören, in der diese Werte verbindlich sind.

Diese Werte, die durch jene Güter erfüllt werden, die von den Mitgliedern einer Wir $_{2}$-Gemeinschaft im Sinne des Gemeinwohls erstrebt werden, stehen untereinander in einer Präferenzordnung, die aufgrund ihrer propositionalen Struktur inferentiell gegliedert ist. Diese inferentielle Ordnung legt fest, welche Dinge, Zustände und Ereignisse wir anderen vorziehen, weshalb Max Scheler in diesem Zusammenhang auch von einer „Vorzugsordnung der Werte" sprach $^{39}$ - wobei die Werte, deren Ordnung und deren Gegebenheitsweise im Rahmen der traditionellen Wertlehren, wie sie im Neukantianismus von Windelband, Rickert und Lask oder im Rahmen der Neuen Ontologie von Wust, Scheler und Hartmann vertreten wurden, freilich nur gegenstandstheoretisch verstanden werden konnten. Im Unterschied zu Rechten, die „uns sagen“, was zu tun ge- oder verboten ist, ,sagen uns" Werte, die sich auf das Gemeinwohl beziehen, also auf das, was aufs Ganze gesehen gut oder besser für uns ist, welches Verhalten sich in bestimmten Kontexten empfiehlt oder nicht empfiehlt, geschätzt oder verabscheut wird,

${ }^{36}$ Weber 1972a, S. 21. Unter „Vergesellschaftung“ versteht Weber dagegen eine soziale Beziehung, bei der „die Einstellung des sozialen Handelns auf rational (wert- oder zweckrational) motiviertem Interessenausgleich oder auf ebenso motivierter Interessenverbindung beruht" (ebd.).

37 Zur Ethik des guten Lebens: Maclntyre 1981; Taylor 1994a; Spaemann 1989; Seel 1995; Wolf 1998.

38 Weber 1972, S. 12.

${ }^{39}$ Scheler 1954, S. 63 f. u. 107 f. 
wichtig oder unwichtig ist. Werte sind also nicht in dem Sinne verpflichtend, wie Rechte die Mitglieder von Rechtsgemeinschaften verpflichten. Werte und Normen sind nicht identisch. Sie lassen sich erstens „durch ihre Bezüge zu verschiedenen Typen des regelgeleiteten oder des zielgerichteten Handelns (unterscheiden); zweitens durch die binäre bzw. graduelle Kodierung ihres Geltungsanspruchs; drittens durch ihre absolute bzw. relative Verbindlichkeit; und viertens durch die Kriterien, denen der Zusammenhang von Norm- bzw. Wertsystemen genügen muß “4. ${ }^{40}$

Zwar wird es oft der Fall sein, daß bei unseren Wertungen, die sich auf das beziehen, was aus der Sicht der Kollektivmitglieder aufs Ganze gesehen gut oder besser für die $\mathrm{Wir}_{2}$-Gruppe ist, auch Zusammengehörigkeitsgefühle im Spiel sind. Dies berechtigt aber nicht schon zu der Schlußfolgerung, daß Wertegemeinschaften Gefühlsgemeinschaften seien oder daß das Werten eine „Gefühlstatsache“ sei. Denn daß „man ein bestimmtes Gefühl hat, kann nie einen hinreichenden Grund abgeben für unsere Achtung vor dem betreffenden Standpunkt, denn das Gefühl kann nicht bestimmen, was bedeutungsvoll ist". ${ }^{41}$ Nonkognitivistische Ansätze, die die Identität von partikularen $\mathrm{Wir}_{2}$-Gemeinschaften direkt auf Gefühle oder Dispositionen zurückführen wollen, verkennen, daß den Gefühlsäußerungen der Geltungsanspruch fehlt, den wir mit „starken Wertungen" und erst recht mit moralischen Urteilen verbinden. ${ }^{42}$ Und diese Ansätze liefern ja auch gar nicht das, was zu liefern sie vorgeben, nämlich eine Erklärung der Vergemeinschaftung von sozialen Beziehungen, sondern eher eine Erklärung der motivationalen Grundlagen solch einer Vergemeinschaftung.

Wertegemeinschaften sind normativ integrierte Gemeinschaften, die sich auf das Gemeinwohl beziehen, welches man mit Taylor als das „Bedeutungsvolle“ einer Wir $_{2^{-}}$ Gemeinschaft bezeichnen kann. Mit der praktischen Frage beziehen wir uns nicht auf subjektive Gefühle oder Entscheidungsgründe (etwa auf das, was für die Mitglieder eines Kollektivs angenehm ist), sondern auf einen objektiven Vorzug, der sich begründen läßt. Wenn wir sagen, daß dies oder jenes ,gut" oder ,besser" für jemanden ist, dann bringen wir damit nicht nur zum Ausdruck, daß wir das, was wir als gut oder besser ansehen, dem, das wir als schlechter ansehen, vorziehen, sondern auch, daß sich unsere Rede auf Gründe und Argumente stützt. Sätze mit „gut" oder „,besser“ prädizieren den Vorzugsanspruch eines Sachverhalts. Es sind Sätze, die einen Begründungsanspruch erheben - im Gegensatz zu Sätzen, mit denen Angaben über einen Zustand gemacht werden, etwa darüber, was den Mitgliedern eines Kollektivs angenehm ist.

Das Problem der Gemeinschaftsbildung ist über intersubjektiv geteilte Werte zu erklären und die Problematik der Werte über unser wertendes Verhalten. Denn spätestens seit Nietzsche und Mill ist klar, daß nicht die Werte der Ursprung des Wertens, sondern umgekehrt ,das Werten der Ursprung der Werte" ist. ${ }^{43}$ Denn wenn wir uns einmal die grammatische Struktur von Wertungen betrachten, dann werden wir feststellen, daß sie

\footnotetext{
${ }^{40}$ Habermas 1996, S. 73.

41 Taylor 1995, S. 46.

${ }^{42}$ Vgl. Habermas 1999, S. 275.

43 Brandom 2000, S. 97.
} 
alle eine implizite Satzstruktur aufweisen. ${ }^{44}$ Wenn aber all unsere Wertungen eine implizite Satzstruktur aufweisen, dann sind Werte, die wir als abstrakte Entitäten verstehen können, die sich einer Nominalisierung von Prädikaten verdanken, über unser wertendes Verhalten aufzuklären, das in Werturteilen seinen Ausdruck findet und Werturteile haben immer eine propositionale Struktur.

Aber auch der kommunitaristische Einwand, der sich auf die von Sandel, MacIntyre oder Taylor gegen den Atomismus vorgebrachten Argumente stützen könnte, spricht nicht gegen den hier unterbreiteten Vorschlag. Denn der methodische Individualismus ist gar nicht auf das verpflichtet, worauf die kommunitaristische Kritik ihn verpflichten will, nämlich auf eine atomistische Sozialontologie. Nach Sandel, MacIntyre und Taylor versteht der methodische Individualismus die Gemeinschaft als eine Ansammlung von Individuen, die durch ihr ,gemeinsames Handeln" Vorteile erhalten wollen, „die sie nicht individuell sichern konnten. Das Handeln ist kollektiv, doch sein Sinn bleibt ein individueller. Das gemeinsame Gute wird ausschließlich durch individuelle Güter gebildet“ ${ }^{45}$ Demgegenüber rekurriert die kommunitaristische Kritik am „ontologisch uninteressierten" Individualismus (der für Sandel, Taylor oder MacIntyre politisch mit dem Liberalismus zusammenfällt) auf ein Gemeinwohl, welches stärker sein soll als das uns vom Individualismus mit seiner atomistischen Ontologie sowie seinem „Begriff vom freien, desengagierten Subjekt" gebotene.

Die kommunitaristische Kritik am methodischen Individualismus setzt also zunächst zwei ungleiche Konsensbildungsprozesse gleich: den diskursiv herbeigeführten Konsens, der auf Gründen beruht, die für alle Beteiligten einsichtig sind und den Konsens, der sich einer Konvergenz der strategischen Kalkulation voneinander unabhängiger Individuen verdankt, die als rationale Egoisten nur ihre eigenen Interessen im Auge haben. Und dann wird auf der Basis dieser Gleichsetzung unterstellt, daß der Rückgriff auf die interpretatorischen Leistungen, das Wollen und die Autonomie des Individuums impliziere, daß das Individuum ein vorsoziales Wesen im Sinne eines „vorsozialen Selbst" sei, so daß sich das Soziale wie im Kontraktualismus nur noch als instrumentelle Kooperation denken läßt. ${ }^{46}$ Dies ist aber überhaupt nicht der Fall. Der methodische Sinn des Rückgangs auf die interpretatorischen Leistungen, das Wollen und die Autonomie des Einzelnen ist ja lediglich der, daß der gemeinschaftlich geteilte Sinn in seinen beiden Dimensionen aus der performativen Einstellung von Kommunikationsteilnehmern erklärbar wird! Die interpretatorischen Leistungen, das Wollen und die Autonomie des Einzelnen sind unter den Bedingungen eines nachidealistischen Denkens die einzigen denkbaren nicht-transzendenten Berufungsinstanzen, von denen her sich der Gemeinsinn in seinen beiden Dimensionen überhaupt verstehen läßt.

Der Dissens zwischen dem methodischen Individualismus und dem Kommunitarismus betrifft noch nicht den ,social character of language“. Und auch die Frage, ob sich „starke Wertungen“, die für das normative Selbstverständnis von partikularen Wir $_{2^{-}}$

44 Das gilt selbst noch für die schlichte Äußerung „Schön!“. Auch eine solche Äußerung, obwohl sie nur aus einem Wort besteht, müssen wir als einen Satz verstehen: nämlich als einen Einwortsatz. Vgl. Schnädelbach 2001, S. 159.

45 Taylor 1994b, S. 112; Sandel 1982, Kap. 2.

46 Tugendhat 1993, S. 202. 
Gruppen konstitutiv sind, vom Kontext einer partikularen Lebensform trennen lassen, ist nicht strittig. Ein Dissens ergibt sich erst bezüglich der Frage der Ontologie und der damit zusammenhängenden Frage, wie das Überindividuelle verstanden werden kann, an dem sich die Gemeinschaftsmitglieder orientieren. Erst hier trennen sich die Wege.

Der methodische Individualismus hält, so gefaßt, konsequent an der hermeneutischen Einsicht fest, daß die Sprache und die Gemeinschaft im Gespräch gründen, das wir sind, so daß er auch das normative Selbstverständnis jener Individuen, die eine Gemeinschaft bilden, aus der Ich-Du-Perspektive von Kommunikationsteilnehmern erklärt. Das kommunitaristische „Wir" jenseits der Ich-Du-Perspektive, mit der das „Wir" gegenüber den performativen Einstellungen von Kommunikationsteilnehmern zu einer dritten Instanz vergegenständlicht wird, betrachte ich als einen grammatisch erzeugten Schein, der aus einer objektivistischen Fehldeutung des Gesprächs resultiert - wobei es natürlich völlig rätselhaft bleibt, wie sich dieses ,Wir" in normativer Hinsicht gegenüber der Ich-Du-Perspektive als vorgängig erweisen lassen soll. ${ }^{47}$

Ich bestreite also nicht die Sinnfälligkeit der Gemeinschaftsorientierung überhaupt, sondern lediglich die These, daß die Gemeinschaft den Individuen logisch voraus liegen soll. Statt dessen behaupte ich, daß die Freiheit des Individuums in und von der Gemeinschaft eine Bedingung dafür ist, daß dieses für eine Gemeinschaft optieren kann. Der methodische Individualismus schließt erstens die auf Heidegger und Wittgenstein zurückgehende These aus, daß es die Sprache sei, die spricht oder daß es die Gemeinschaft sei, die bestimmte sprachliche Handlungen ,als richtig ansieht", da in einer solchen Sprachontologie die hermeneutischen Leistungen von den Kommunikationsteilnehmern entweder auf die Sprache übertragen werden, die dann den Platz eines subjektlosen Erzeugers von Sinn einnimmt oder aber auf die Gemeinschaft, die dann das tun soll, was für gewöhnlich nur Individuen tun können. Und der methodische Individualismus schließt zweitens die an Hegel orientierte These aus, daß, ,die Individuen [...] eine supraindividuelle Entität anerkennen, die in sich wertvoll ist und von der sie, die Individuen, erst ihren Wert beziehen sollten" ${ }^{48}$ da diese Gemeinschaftsontologie die These von der Priorität des Sozialen nur mit Rekurs auf transzendente Prämissen begründen kann, gegen die nicht nur philosophische, sondern auch empirische Argumente sprechen.

Weder die kommunitaristische Sprachontologie noch die kommunitaristische Gemeinschaftsontologie vermag zu überzeugen, da im ersten Fall die interpretatorischen Leistungen und im zweiten Fall das Wollen und die Autonomie der Individuen auf der Strecke bleiben. Zwar hat der Kommunitarismus gegenüber dem Atomismus darin recht, daß die Wir-Perspektive im Gespräch gründet und sich dementsprechend nicht aus einer Aufstockung von unabhängigen Ich-Perspektiven erklären läßt. ${ }^{49}$ Und er behält gegenüber dem Atomismus auch noch darin recht, daß ,auf der Grundlage einer kooperativen Vorstellung von Gemeinschaft [...] unklar (bleibt), wie das moralische

\footnotetext{
47 Aus einer differenztheoretischen Perspektive sind ähnliche Einwände von Seyla Benhabib (1993) und Iris M. Young (1990) erhoben worden.

48 Tugendhat 1993, S. 203.

49 Vgl. Taylor 1986b, S. 41.
} 
Fundament dieser Gemeinsamkeit aussehen soll “ ${ }^{50}$ Von daher hat das auf Aristoteles zurückgehende kommunitaristisch-ethische Gemeinschaftsverständnis gegenüber dem auf Locke zurückgehenden individualistisch-instrumentalistischen Gemeinschaftsverständnis den Vorzug, daß es die Gemeinschaft nicht nach dem Organisationsmodell konzipieren muß, sondern nach dem Modell der Zugehörigkeit zu einer sich selbst bestimmenden ethisch-kulturellen Gemeinschaft beschreiben kann.

Der Kommunitarismus neigt jedoch dazu, die Wir-Haltung gegenüber der Ich-DuPerspektive als vorgängig zu betrachten, so daß Wir ${ }_{2}$-Gemeinschaften als Subjekte größeren Formats erscheinen. Und genau hierin liegt das Problem: Kommunitaristen sprechen über die Gemeinschaft, als ob sie so handeln könnte, wie die einzelnen Gemeinschaftsmitglieder. Dies ist aber nicht der Fall. Nicht die Gemeinschaft handelt, spricht oder beurteilt, sondern dies tun bekanntlich immer nur ihre Mitglieder. Von daher sind auch die Redeweisen von einem „gemeinschaftlichen Handeln“, von einem „gemeinschaftlichen Sprechen" oder von einem „gemeinschaftlichen Beurteilen“, entweder Metaphern, die man erst noch ausbuchstabieren müßte, oder aber Fiktionen, mit denen die Gemeinschaft unzulässig personifiziert wird.

\section{Gemeinsinn und praktische Identität}

Sprach- und vernunftbegabte Wesen, also Mitglieder der universalen Wir - $^{-}$ Gemeinschaft, sind also dann Mitglieder einer partikularen $\mathrm{Wir}_{2}$-Gemeinschaft, wenn sie sich an der Konzeption des Guten einer solchen Gemeinschaft orientieren - womit sie gleichzeitig zu erkennen geben, daß sie einen Gemeinsinn haben. Gemeinsam geteilte Überzeugungen sind nicht nur für ein wechselseitiges Verstehen relevant, ohne welches es überhaupt keine Wir $_{1}$-Gemeinschaft geben würde, sondern auch für die Konstitution von $\mathrm{Wir}_{2}-\mathrm{Gemeinschaften}$ in einem engeren Sinne. Solche $\mathrm{Wir}_{2}$-Gemeinschaften beziehen sich auf einen Korpus von Überzeugungen, der als kollektiv verbindlicher und

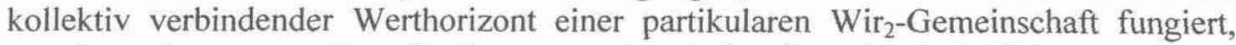
womit $\mathrm{zu}$ den notwendigen Bedingungen einer jeden Gemeinschaftsbildung nun auch die hinreichenden Bedingungen benannt wären.

Relevant ist hier nicht, daß sich „uninteressante“ oder „dünne Überzeugungen“ vor jedermann und ,interessante " oder „dicke Überzeugungen“" nur innerhalb der eigenen $\mathrm{Wir}_{2}$-Gemeinschaft rechtfertigen lassen. Der entscheidende Punkt ist vielmehr, daß diejenigen Überzeugungen, die relativ auf das normative Selbstverständnis einer partikularen $\mathrm{Wir}_{2}$-Gruppe sind, Überzeugungen sind, die das eigene ,Zu-Sein ${ }^{651}$ der Gruppe betreffen. Es ist also nicht schon die sprachliche Kommunikation als solche, die sich auf die Identität einer partikularen $\mathrm{Wir}_{2}$-Gemeinschaft bezieht oder für diese bürgt, sondern erst die, die sich auf das Zu-Sein des jeweils eigenen Kollektivs bezieht.

Im Anschluß an Aristoteles, der als erster den aktivischen Aspekt im Sichverhalten zum eigenen Sein herausgearbeitet hat, haben Heidegger, Tugendhat und Taylor gezeigt, daß ein praktischer Selbstbezug erst dann vorliegt, wenn die Kommunikation das

50 Sandel 1994, S. 28.

si Vgl. Heidegger 1979, S. 36. 
je eigene Zu-Sein betrifft, was immer dann der Fall ist, wenn es in dieser Kommunikation um die praktische Frage geht, was aus der Perspektive der ersten Person Plural aufs Ganze gesehen gut oder besser für „uns“ ist. Es ist erst dieser Bezug auf einen gemeinsam geteilten Werthorizont, der die praktische Identität von partikularen $\mathrm{Wir}_{2}$ Gemeinschaften verbürgt. Die Mitglieder von partikularen Wir $_{2}$-Gemeinschaften stellen also nicht nur die Frage, was zur Erhaltung des Lebens gut ist. Sie stellen darüber hinaus die Frage nach dem guten Leben, ${ }^{52}$ wobei hier der praktische Bezug zum je eigenen Sein der letzte Bezug allen Wollens ist.

Der Bezug zum je eigenen $\mathrm{Zu}$-Sein ist erst dann gegeben, wenn das Gespräch einen praktischen Selbstbezug bekommt, wenn also die Gesprächsteilnehmer überlegen, was sie jetzt und in der Zukunft tun wollen. Wenn man mit Heidegger, Tugendhat und Taylor unterstellt, daß man sich in allem Sichverhalten zu den jeweils eigenen Handlungsmöglichkeiten verhält, kann man auch sagen, daß sich eine Gruppe dann zu sich verhält, wenn ihre Mitglieder zu ihrem Tun als Gruppe Stellung beziehen, wie man sich auch erst dann zu einer anderen Gruppe verhält, wenn man zu deren Tun Stellung bezieht. ${ }^{53}$ In beiden Fällen ist der relevante Maßstab das jeweilige Gemeinwohl der Gemeinschaft. Ein praktischer Selbstbezug liegt demnach noch nicht vor, wenn wir mit anderen über irgend etwas reden. Und er liegt auch nicht schon dann vor, wenn wir mit uns selbst sprechen - im Falle von Gruppen könnte man hier etwa an die Versammlung einer Dorfgemeinschaft denken, in der die Dörfler über die Frage debattieren, ob ihr Dorf das schönste im Kreis ist oder an die Europäische Union, in der Europäer darüber streiten, wie krumm Bananen sein dürfen, um auf dem europäischen Markt verkauft werden zu können. Solche Debatten konstituieren allenfalls ein „,commercium“, aber keine „communio", also eine über gemeinsam geteilte Werte integrierte Gemeinschaft, die ein praktisches Selbstverständnis gemeinsam teilt.

Kontingent zusammengewürfelte reale Kommunikationsgemeinschaften sind zwar Gemeinschaften, die sich sowohl aus der Teilnehmer- als auch aus der Beobachterperspektive von anderen Gemeinschaften abgrenzen lassen, beispielsweise von denen, die gerade nicht miteinander über etwas sprechen, so daß die Mitglieder dieser Kommunikationsgemeinschaft ebenfalls ,wir" sagen könnten. Diese Wir ${ }_{2}$-Gruppe hätte aber nicht die Identität, die man als qualitative Identität bezeichnen kann. Das Kollektiv wäre daher auch nur numerisch, nicht aber qualitativ identifizierbar. Zwar ist eine gemeinsame Kommunikation eine notwendige Voraussetzung dafür, daß Kollektive auch unter qualitativen Gesichtspunkten identifizierbar sind. Sie darf jedoch nicht schon mit dieser gleichgesetzt werden. Erst wenn diese Kommunikation das betrifft, was das Kollektiv künftig tun will, verhalten sich seine Mitglieder als Kollektiv selbstreflexiv zu ihrem Tun.

Partikulare $\mathrm{Wir}_{2}$-Gemeinschaften teilen zwei Varianten des „Gemeinsinns“: Einerseits sprachlich geteilte Bedeutungen und andererseits bestimmte Wertüberzeugungen,

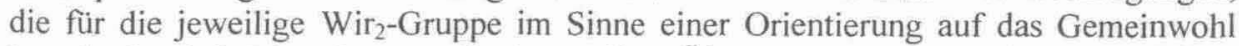
konstitutiv sind. Diese kommunikativ geteilten Überzeugungen bezüglich der kollektiv anerkannten Werte sind konstitutiv für die praktische Identität der Gemeinschaft - wes-

52 Vgl. Aristoteles 1979, I 6.

${ }^{33} \mathrm{Vgl}$. Tugendhat 1979, S. 160. 
halb sich der Begriff des "Gemeinsinns" im zweiten Fall auch nicht auf alle und jeden bezieht, sondern eben nur auf die Mitglieder jener Gemeinschaften, die einen Sinn im Sinne der Orientierung auf das Gemeinwohl teilen.

\section{Vergangenheit und Zukunft}

In bezug auf den Gemeinsinn im Sinne sprachlich geteilter Bedeutungen wurden bereits drei Punkte fixiert: Erstens, daß dieser Sinn die Möglichkeiten des Mein- und Verstehbaren eröffnet und begrenzt, so daß innerhalb dieser Möglichkeiten viel, aber eben „doch nicht alles möglich“ ist; ${ }^{54}$ zweitens, daß dieser Sinn sich im Ge- oder Mißlingen der sprachlichen Kommunikation bildet und erhält; und drittens, daß es daher auch keinen dem performativen Sein der Sprache entzogenen externen Maßstab solchen Geoder Mißlingens geben kann. ${ }^{55}$ Was es hier gibt, sind immer nur die internen Korrektive der sprachlichen Praxis selbst.

$\mathrm{Zu}$ allen drei Punkten gibt es für den Gemeinsinn im Sinne der Orientierung auf das Gemeinwohl einer partikularen $\mathrm{Wir}_{2}$-Gemeinschaft eine Entsprechung, insofern wir erstens feststellen können, daß auch dieser Sinn Möglichkeiten eröffnet und begrenzt, nämlich die eines Verhaltens, welches sich in bestimmten Kontexten empfiehlt oder nicht empfiehlt, geschätzt oder verabscheut wird, wichtig oder unwichtig ist, so daß für die Gemeinschaftsmitglieder innerhalb dieser Möglichkeiten viel, aber eben nicht alles möglich ist; zweitens, daß sich auch dieser Sinn im Ge- oder Mißlingen der sprachlichen Kommunikation bildet und erhält; und drittens, daß es auch hier keinen dem performativen Sein der Sprache entzogenen externen Maßstab eines solchen Ge- oder Mißlingens geben kann, da es auch hier immer nur die internen Korrektive der sprachlichen Praxis selbst gibt.

Man kann daher sagen: Hier wie dort muß sich das Gelingen sprachlicher Kommunikation im Zusammenhang des Lebens bewähren. Während sich jedoch im ersten Fall diese Kommunikation und ihr Gelingen nicht partikularistisch verstehen lassen, da sie an das Forum einer prinzipiell nicht begrenzten Wir ${ }_{1}$-Gemeinschaft im Sinne aller jener Wesen gebunden sind, denen wir Vernunft unterstellen, müssen diese Kommunikation und ihr Gelingen im anderen Fall partikularistisch verstanden werden, da sie an das Forum einer prinzipiell begrenzten $\mathrm{Wir}_{2}-\mathrm{Gemeinschaft} \mathrm{im} \mathrm{Sinne} \mathrm{all} \mathrm{jener} \mathrm{vernunftbe-}$ gabten Wesen gebunden ist, denen es in ihrem Sein um das Wohl ihrer partikularen Gemeinschaft selbst geht.

Von daher ist vernünftigerweise damit zu rechnen, daß wir bei „Auseinandersetzungen zwischen Gemeinschaften über die richtige Lebensweise“ auf tiefgreifende „Unvereinbarkeiten" stoßen. ${ }^{56}$ Denn die Frage, ob sich alternative Lebensentwürfe von partikularen Wir $_{2}$-Gruppen auf einer neutralen Basis entscheiden lassen, die sich auf gemeinsam geteilte Überzeugungen gründet, müssen wir negativ beantworten. Zwar stehen die Mitglieder aller partikularen $\mathrm{Wir}_{2}$-Gruppen im „logischen Raum der Grün-

${ }^{54}$ Gadamer 1990, S. 273.

55 Wellmer 1995, S. 137.

56 Vgl. MacIntyre 1994, S. 89. 
$\mathrm{de}^{\text {"* }}$, in einem Raum also, von dem ich sagte, daß er sich nicht partikularisieren lasse. Dieser Raum, den , Wir $_{1}{ }^{\text {" }}$ als rationale Wesen bewohnen, ist aber nicht deckungsgleich mit dem Raum, den ,Wir ${ }^{\prime}$ als Mitglieder von partikularen $\mathrm{Wir}_{2}$-Gemeinschaften bewohnen. Wir begegnen hier einem Partikularismus, der konfligierende Überzeugungen bezüglich der Frage, was aufs Ganze gesehen gut oder besser für die jeweilige Gemeinschaft ist, als eine notwendige Bedingung der Möglichkeit dafür erkennt und anerkennt, $\mathrm{da} ß$ es überhaupt verschiedene Gemeinschaften gibt. Wirkliche $\mathrm{Wir}_{2}$-Gemeinschaften können gar nicht anders als verschieden sein. ${ }^{57}$

Daher gilt: „Die Rationalität eines selbstbestimmten Lebens ist in einem bestimmten Sinn relativ“68 ${ }^{65}$, nämlich relativ zu dem Kollektiv, dem es in seinem Sein um dieses selbst geht. Und sie bezieht sich auf ein Gutes, das sich im Fall von partikularen Wir $_{2}$ Gemeinschaften als Gemeinwohl verstehen läßt. Ein ,angemessener Begriff der praktischen Vernunft", der sich auf die Rationalität eines selbstbestimmten Lebens bezieht, muß daher „kontextabhängig sein. Denn ein solcher Begriff folgt nicht aus formalen Prinzipien, die von praktischen Handlungszusammenhängen unabhängig sind, sondern zeichnet sich durch eine extensive Explikation dessen, was Handlungskontexte implizieren, aus". 59

Ich möchte an dieser Stelle nicht die These diskutieren, ob sich die praktische Vernunft wirklich nur als eine „extensive Explikation“" von Hintergrundvoraussetzungen verstehen läßt - was man sicher bestreiten kann, da die praktische Vernunft auf diese Weise zwar nicht falsch, ganz sicher aber unterbestimmt wäre. Nicht bestreitbar scheint mir indes, daß sich die Rationalität eines selbstbestimmten Lebens zunächst nicht auf unser theoretisches Wissen, sondern auf unser praktisches Wissen bezieht, auf ein Wissen also, das ,das Richtigleben im ganzen betrifft “ - und damit auf ein Kollektiv, das sich praktisch zu sich verhält. ${ }^{60}$ Denn offensichtlich ist die Frage, was aufs Ganze gesehen gut oder besser für die jeweilige Gemeinschaft ist, keine theoretische Frage, deren Antwort in einer deskriptiven Aussage bestehen könnte, sondern eine praktische Frage, die ihre Antwort nur in einem Satz finden kann, der eine Absicht oder einen Entschluß zum Ausdruck bringt. Das Sein, auf das die Fragenden mit dieser Frage Bezug nehmen, ist nicht die abgelaufene Existenz der Gruppe, also ihre gemeinsam geteilte Geschichte, die sich nur noch konstatieren ließe, sondern die jeweils bevorstehende Existenz, also ihre gemeinsam zu teilende Zukunft, die die $\mathrm{Wir}_{2}$-Gruppe auf die eine oder andere Art vollziehen oder nicht vollziehen muß. ${ }^{61}$

Zwar ist klar, daß die gemeinsam geteilte Geschichte bei der Beantwortung von praktischen Fragen schon insofern eine ganz wesentliche Rolle spielt, als es ja gerade diese Geschichte ist, die den Kontext bildet, in dem sich die Identität von partikularen $\mathrm{Wir}_{2^{-}}$ Gemeinschaften bildet - weshalb diese auch zu ihrer Geschichte keine hypothetische Einstellung einnehmen können. Von daher kann deren kollektives Selbstverständnis nicht unabhängig von der Geschichte verstanden werden, die die Mitglieder von $\mathrm{Wir}_{2-}$

\footnotetext{
57 Vgl. Walzer 1996, S. 197.

58 Seel 1995, S. 128

59 Taylor 1986a, S. 131.

${ }^{60}$ Gadamer 1990, S. 326

61 Vgl. Tugendhat 1979, S. 177.
} 
Gemeinschaften teilen. Und klar ist auch, daß diese Geschichte verfehlt werden kann, nämlich dann, wenn die hermeneutischen Rekonstruktionen ein falsches oder einseitiges Bild von dieser Geschichte entwerfen, so daß auch die Antwort auf die praktische Frage eine Antwort sein wird, die man dann kaum als adäquat bezeichnen könnte.

Daher ist jede Antwort auf die praktische Frage auf eine authentische, kontextuell angemessene und kohärente hermeneutische Rekonstruktion der geschichtlichen Voraussetzungen des gegenwärtigen Wünschens und Hoffens der Mitglieder von $\mathrm{Wir}_{2^{-}}$ Gruppen angewiesen. Denn wenn sich unsere Orientierungen auf epistemischen Irrtümern, Täuschungen und Wunschvorstellungen über uns und die Welt gründen, dann ist kaum damit zu rechnen, daß die Handlungen, die jene Absichtssätze wahr machen sollen, diese auch wirklich wahr machen werden. Entgegen der Behauptung des konstruktivistischen Narrativismus, der zufolge alles Beliebige erzählt werden kann, behaupte ich, daß die Antwort auf die praktische Frage etwas mit Kriterien zu tun hat, die sicher viel, die deshalb aber eben noch lange nicht alles als möglich erscheinen lassen. Man kann nicht von allem und jedem jede beliebige Geschichte erzählen, zumindest kann man dies nicht, ohne daß der Narrativismus in einen extremen Idealismus umschlägt, der unter Kohärenzgesichtspunkten kaum als plausibel gelten kann.

Jede hermeneutische Rekonstruktion der Identität von partikularen Wir $_{2}$-Gruppen muß sich in einsichtsvoller Weise der Quellen des Wir versichern, von Quellen, die in gewisser Hinsicht immer auch Quellen des Selbst sind - wobei hier wie dort die Pluralform deutlich machen soll, daß es sich bei der Identitätsbildung von Individuen und Gruppen nicht nur um eine Quelle handelt, aus der das normative Selbstverständnis von Gruppen und Individuen zu erklären ist, sondern um eine Vielzahl von Quellen, so daß wir hier in Analogie zu einem ,post-sozialen Selbst “62 ${ }^{46}$ von einem ,post-sozialen Wir ${ }_{2}{ }^{*}$ sprechen können - wobei „post-sozial“ nicht im Sinne einer Unabhängigkeit von sozial geprägten Identitäten, Lebensformen und Traditionen zu verstehen ist, sondern im Sinne einer reflexiven Distanz zu partikularen Identitäten, Lebensformen und Traditionen, also im Sinne einer Tradition zweiter Ordnung, so wie sie dem normativen Selbstverständnis moderner Gesellschaften entspricht.

Diese hermeneutische Rekonstruktion einer gemeinsam geteilten Geschichte, die adäquat oder weniger adäquat, kohärent oder weniger kohärent, angemessen oder weniger angemessen ausfallen kann, ist eine notwendige Bedingung dafür, daß die praktische Frage eine Antwort findet. Sie ist aber eben nur die Voraussetzung einer solchen Antwort und nicht schon diese selbst. Auf dem Weg der hermeneutischen Rekonstruktion bekommt man immer nur eine Antwort auf die Frage, wer wir in der Vergangenheit waren, nicht aber auf die Frage, wer wir künftighin sein wollen. Die abschließende Antwort auf die praktische Frage besteht immer in einem Absichtssatz - im Gegensatz zur Antwort auf eine theoretische Frage, die stets eine Behauptung sein wird.

Selbstverständigungsdiskurse unterscheiden sich darum grundsätzlich von anderen Diskursen. Beschreibungen und Neubeschreibungen, die sich auf die objektive Welt beziehen, verwenden wir, um wahre Aussagen über Dinge und Ereignisse zu machen. Beschreibungen und Neubeschreibungen, die sich auf die soziale Welt einer partikularen $\mathrm{Wir}_{2}$-Gruppe beziehen, verwenden wir hingegen, um unserem Wunsch nach einer

\footnotetext{
${ }^{62}$ Vgl. Wellmer 1993, S. 177.
} 
Veränderung der eigenen gruppenspezifischen Existenzweise Ausdruck zu verleihen wobei wir durch unser eigenes Tun dafür Sorge tragen müssen, daß ein Prädikat auf die künftige Existenzweise der Gruppe zutrifft, das auf ihre frühere Existenzweise noch nicht zutraf. Der Unterschied zwischen einer revisionären Neubeschreibung eines Gegenstandsbereiches in der objektiven Welt und einer revisionären Neubeschreibung der partikularen $\mathrm{Wir}_{2}$-Gruppe ist wichtig. Denn er macht deutlich, daß Kollektivmitglieder, die sich mit dem Ziel neu beschreiben, ein anderes Kollektiv zu werden, mit dieser Neubeschreibung erstens einen normativen Maßstab errichten, an den sich die Mitglieder selbst binden wollen und an dem sich daher auch ihre tatsächliche Existenzweise „, immanent" messen und beurteilen läßt; und zweitens sorgen sie mit dieser Neubeschreibung dafür, daß ein Prädikat auf ihr derzeitiges Kollektiv zutrifft, das auf ihr früheres Kollektiv noch nicht zutraf.

Wenn beispielsweise die Mitglieder eines Kollektivs dieses als feige, als opportunistisch oder als antidemokratisch beschreiben, wenn also aus der Perspektive der ersten Person Plural eine kollektive Selbstbeschreibung so ausfällt, daß die Kollektivmitglieder selbst sagen „Wir sind $x^{\prime}$, und wenn diese Beschreibung zudem dazu führt, daß sich das Kollektiv ändert, so daß künftighin weder aus der Teilnehmer- noch aus der Beobachterperspektive dieses Kollektiv als feige, opportunistisch oder antidemokratisch bezeichnet werden kann, dann haben die Mitglieder der entsprechenden $\mathrm{Wir}_{2}$-Gruppe dafür Sorge getragen, daß von nun an ein Prädikat auf sie zutrifft, daß vormals noch nicht auf sie zutraf. Im Unterschied zu umfassenden Neubeschreibungen des Sonnensystems, die, sofern sie wahr sind, immer wahr sind, werden diese Neubeschreibungen von „uns" durch unser eigenes Tun „wahr gemacht". Nicht schon die Neubeschreibung allein sorgt also für eine Veränderung der individuellen Existenzweise einer partikularen $\mathrm{Wir}_{2}$-Gruppe, sondern erst die Neubeschreibung in Verbindung mit einer Handlung. Durch die Handlung muß das Kollektiv dafür Sorge tragen, daß es sich selbst im Sinne der Neubeschreibung verändert, so daß künftighin die Existenzweise der eigenen Beschreibung ,entspricht".

Im Fall des Gemeinwohls betrifft die praktische Frage in der ersten Person Plural immer das gemeinsame Handeln, das Tun und das Leben; sie betrifft immer die unmittelbare oder weitere gemeinsame Zukunft des Kollektivs; sie betrifft den Spielraum der freien Entscheidung - denn wenn dieser Spielraum klar wäre, müßte ja gar nicht gefragt werden -; sie betrifft die Grenzen der freien Entscheidung - denn wo nichts vorgegeben wäre, müßte nicht überlegt werden -; sie betrifft die Möglichkeit, diese Frage überhaupt zu stellen oder sie zu verdrängen; und sie betrifft schließlich das, was aufs Ganze gesehen gut oder besser für ein Kollektiv ist. ${ }^{63}$

Ein rechtverstandener Partikularismus bezieht sich also nicht auf Überzeugungen schlechthin, sondern ausschließlich auf jene, die konstitutiv für ein praktisches Selbstverständnis einer $\mathrm{Wir}_{2}$-Gruppe sind, das sich nicht vom Kontext einer partikularen Lebensform abtrennen läßt. Die Frage ist nicht, was gleichermaßen gut oder besser für alle ist, sondern was jeweils gut oder besser für die Mitglieder einer partikularen $\mathrm{Wir}_{2}$ Gruppe ist, der es in ihrem Sein um dieses selbst geht. Ethische Fragen der Selbstverständigung sind Fragen, mit denen sich die Mitglieder von partikularen $\mathrm{Wir}_{2}$-Gruppen

\footnotetext{
${ }^{63} \mathrm{Vgl}$. Tugendhat 1979, S. $194 \mathrm{f}$.
} 
in der Perspektive der ersten Person Plural auf alternative kollektive Handlungsmöglichkeiten im Sinne des Gemeinwohls beziehen, wobei die Gruppenmitglieder sich ihrer Identität vergewissern und wissen wollen, welches Leben sie gemeinsam im Lichte dessen, wer sie sind und künftighin sein möchten, führen sollen.

\section{Gemeinwohl, Gemeinschaft und Identität}

Fragen des Gemeinwohls sind ethische Fragen, die für das normative Selbstverständnis von partikularen $\mathrm{Wir}_{2}$-Gemeinschaften konstitutiv sind. Wenn wir der deontologischen Abgrenzung von Moral und Ethik folgen, wie sie im Anschluß an Kant im Umfeld prozeduralistischer Ansätze vertreten wird, dann können wir ethische Fragen als die Fragen bezeichnen, die sich auf eine Konzeption des guten oder besser: des nicht-verfehlien Lebens beziehen lassen. Ethische Fragen bemessen sich nicht an moralischen Gesichtspunkten, sondern an dem, was Charles Taylor "starke Wertungen" nennt, die intern mit dem normativen Selbstverständnis einer partikularen $W_{i r}$-Gruppe verschränkt sind. Diese interne Verschränkung ist gleichzeitig der Grund dafür, daß sich diese Fragen nicht vom Kontext bestimmter Lebensformen trennen lassen, da es hierbei ausschließlich darum geht, was aus der Sicht der Gruppenmitglieder dieser Wir $\mathbf{W}_{2}$-Gruppe aufs Ganze gesehen gut oder besser für sie ist.

Gemeinsam geteilte Überzeugungen bezüglich der Wertmaßstäbe gehören zu den Gemeinsamkeiten, die jene, die sie teilen, miteinander verbinden und als Mitglieder einer partikularen $\mathrm{Wir}_{2}$-Gemeinschaft ausweisen. „Daß sie für uns ethisch gut sind, heißt, daß die Orientierung an ihnen uns in solche Beziehungen zueinander setzt oder hält, in denen zu stehen für jeden von uns Bestandteil seines normativen Selbstverständnisses ist; die Orientierung an solchen Maßstäben bringt Ego uno actu in Übereinstimmung mit sich und mit Alter als Angehörige einer Gemeinschaft in Übereinstimmung. " ${ }^{64}$ Was immer die Mitglieder partikularer $\mathrm{Wir}_{2}$-Gruppen darüber hinaus noch teilen mögen, sie teilen in jedem Fall die Wertmaßstäbe und Überzeugungen, die in der Gemeinschaft verbindlich sind. Sie sind es, die die Gemeinschaft in Übereinstimmung mit sich bringen, so daß wir sagen können: Mitglied einer Gemeinschaft zu sein heißt daher, jemand zu sein, der die ,praxisimpliziten Normen der Gemeinschaft befolgen sollte. Mitgliedschaft in einer Gemeinschaft hat diese normative Signifikanz; sie ist ein normativer Status.“ Der Status des Gemeinschaftsmitglieds hat normative Konsequenzen, insofern Mitglieder von Gemeinschaften bestimmten Richtigkeitsstandards unterworfen sind. In dieser Hinsicht ist die „Gemeinschaftszugehörigkeit [...] ein Begriff, der so gebraucht wird, daß er normative Folgen der Anwendung nach sich zieht, nämlich daß sich das Mitglied vor den Urteilen der Gemeinschaft verantworten muß, daß es ihrer Autorität unterworfen ist". ${ }^{65}$

Ich habe gezeigt, daß dies Rationalität in einem basalen Sinne voraussetzt, der sich nicht partikularistisch verstehen ließ. Denn nur Wesen, denen wir in diesem basalen Sinne Rationalität zuschreiben, sind Wesen, die sich vor den Urteilen der Gemeinschaft

\footnotetext{
64 Wingert 1993, S. 146.

65 Brandom 2000, S. 85 f.
} 
für das, was sie denken und tun, verantworten können. Gleichwohl ist jene Vernünftigkeit, die darin zum Ausdruck kommt, daß wir in diesem basalen Sinne von Rationalität „wir ${ }_{1}$ "sagen, nicht mit jener Vernünftigkeit identisch, die man seit Aristoteles die praktische nennt. Daß , wir ${ }_{1}$ " gemeinsam im Raum der Gründe stehen, indem wir Gründe für unsere Einstellungen und Verhaltensweisen geben, kennzeichnet „uns “ “ als rationale Wesen einer universellen Wir $_{1}$-Gemeinschaft. Daß einige von ,uns " “ aus dieser univer-

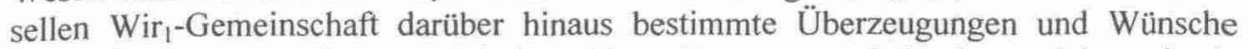
teilen, die das ausmachen, was ich das ethisch Gute genannt habe, kennzeichnet sie als rationale Wesen einer im sozialen Raum und in der historischen Zeit verorteten partikularen $\mathrm{Wir}_{2}$-Gemeinschaft.

Ethische Überzeugungen sind kontextabhängig - und damit ,nicht öffentlich“ in dem Sinne, daß alle Menschen ihnen zustimmen müßten. Denn das ethisch Gute ist stets an ein Sich-selbst-Verstehen derjenigen gebunden, die sich als Mitglieder einer partikularen $\mathrm{Wir}_{2}$-Gruppe praktisch zu sich verhalten. Das ethisch Gute ist relativ zu einer konkreten Gruppe, da es unabtrennbar zu ihrer Existenzweise gehört - was nach Habermas der Grund dafür ist, daß „ethischen Fragen [...] die Referenz zur ersten Person und damit der Bezug zur Identität [...] einer Gruppe grammatisch eingeschrieben" ist. ${ }^{66}$

Nun ist aber diese „Referenz zur ersten Person" Plural noch keine Besonderheit ethischer Fragen. Denn auch hermeneutischen oder moralischen Fragen wäre diese „Referenz zur ersten Person“ "Plural ,grammatisch eingeschrieben" - nämlich in Gestalt der Präsupposition „Wir ${ }_{1}$ sind vernünftig“. Ein Charakteristikum ist erst der Bezug auf die gemeinsam geteilten Überzeugungen vom guten Leben jener, die sich als Mitglieder einer partikularen $\mathrm{Wir}_{2}$-Gruppe verstehen. Denn nur dann bezieht sich das „Wir“ ja auf eine $\mathrm{Wir}_{2}$-Gruppe, die kleiner ist als die gesamte Menschheit. Nur im ethischen Fall bezieht sich das "Wir“ auf ein Kollektiv, das ein gemeinschaftliches Leben führt. Kollektive Identitäten bilden sich in Gestalt eines normativen Konsenses, der die Frage des Gemeinwohls betrifft, die Frage, was aufs Ganze gesehen gut oder besser für die Mitglieder einer partikularen $\mathrm{Wir}_{2}$-Gruppe ist - wobei sich die Identität der Gruppenangehörigen gleichursprünglich mit der Identität der Gruppe herstellt.

In beiden Fällen kann der Begriff der Identität hermeneutisch gerechtfertigt werden. ${ }^{67}$ Die symbolischen Strukturen, die für die Einheit des Kollektivs und seiner einzelnen Angehörigen konstitutiv sind, stehen nämlich in einem internen Zusammenhang mit der Verwendung der Personalpronomen, also jener indexikalischen Ausdrücke, die für Zwecke der Identifizierung von Personen und Gruppen gebraucht werden. Ich behaupte also zweierlei: Ich behaupte erstens, daß diese Identitätsbedingungen auf den Begriff des „Gemeinwohls" und damit auf den „Gemeinschaftsbegriff“ verweisen, insofern diese kollektive $\mathrm{Wir}_{2}$-Identität auf eine nicht-kontingente Weise mit bestimmten Wertungen und damit mit bestimmten Überzeugungen und Wünschen verknüpft ist, die sich auf das beziehen, was aufs Ganze gesehen gut oder besser für die Wir ${ }_{2}$-Gruppe ist. Dabei steht der Begriff der "Identität" in diesem Zusammenhang für die „Fähigkeit" eines Kollektivs, sich auf der Grundlage eines reflektierten Selbstverhältnisses als das-

\footnotetext{
${ }^{66}$ Vgl. Habermas 1996, S. 252.

67 Tietz 2002b, Kap. 4.
} 
jenige zu identifizieren, das es sein will. ${ }^{68}$ Und ich behaupte zweitens, daß dieses von den Mitgliedern des Kollektivs gemeinsam geteilte Welt- und Selbstverständnis in Form gemeinsam geteilter Überzeugungen und Wünsche, das für die Einheit der Wir $2^{-}$ Gruppe und deren Angehörige konstitutiv ist, in einem notwendigen Zusammenhang mit der Verwendung der Personalpronomina steht, also mit dem System jener indexikalischen Ausdrücke, mit denen Personen auf sich, auf andere Personen oder aber auf Personengruppen Bezug nehmen.

Wie dieser Bezug genau zu verstehen ist, läßt sich anhand Verwendungsweise des Wortes ,wir" klären. Der Ausdruck ,wir" ist ein Personalpronomen. Er gehört mit den übrigen Personalpronomina, den Orts- und Zeitadverbien und den Demonstrativa zur Klasse der kontextabhängigen Ausdrücke. Diese bilden zusammen mit den Namen und den Kennzeichnungen die Klasse der singulären Termini, mit denen Sprecher einzelne Gegenstände identifizieren. „Ein singulärer Terminus ist ein Ausdruck, dessen Funktion es ist, einen einzelnen Gegenstand zu bezeichnen. Genauer muß man sagen: die Funktion eines singulären Terminus besteht darin, daß ein Sprecher mit ihm angibt, welchen von allen Gegenständen er meint und d. h.: von welchem von allen Gegenständen der den singulären Terminus in einem ganzen Satz ergänzende Prädikatausdruck gelten soll."69

Wie die übrigen deiktischen Ausdrücke erhalten Personalpronomina nur in dem jeweiligen Verwendungskontext einen eindeutigen Sinn. Ein Sprecher, der den Ausdruck „wir" verwendet, gibt damit zu erkennen, daß er sich als Mitglied einer bestimmten $\mathrm{Wir}_{2}$-Gruppe versteht - zumindest wenn er dies nicht ironisch meint oder lügt. Mit dem Ausdruck ,wir" nimmt ein Sprecher auf ein Kollektiv von Menschen Bezug, das sich unter bestimmten Gesichtspunkten von anderen Kollektiven unterscheiden läßt und dem er sich selbst zugehörig zählt, was ihm freilich von anderen Kollektivmitgliedern bestritten werden kann, so daß der Sprecher nun das Wort ,ihr" verwenden muß, wenn er sich weiterhin auf dieses Kollektiv beziehen will.

Dieses Ergebnis erscheint mir nicht trivial. Denn es zeigt, daß der Status der Zugehörigkeit ein Status ist, der zugeschrieben wird. Genauer: Der Status der Zugehörigkeit zu einer partikularen $\mathrm{Wir}_{2}$-Gemeinschaft ist ein Status, den wir uns wechselseitig zuschreiben - wobei wir auch wechselseitig davon ausgehen, daß wir uns die Gruppenzugehörigkeit wechselseitig zuschreiben. Der Status der Zugehörigkeit ist keine Naturtatsache, sondern ein soziales Faktum, das anerkennungstheoretisch aus der diskursiven $\mathrm{Zu}$ schreibungspraxis von sprach- und handlungsfähigen Subjekten erklärt werden muß. Zuschreiben heißt, eine Festlegung einem anderen zuzuerkennen und selbst eine Festlegung von anderen anzuerkennen. Ohne diese wechselseitige Zuschreibungspraxis gäbe es nicht nur keine verschiedenen $\mathrm{Wir}_{2}$-Gruppen, sondern noch nicht einmal eine einzige $\mathrm{Wir}_{2}$-Gruppe. Der Status der Zugehörigkeit zu partikularen $\mathrm{Wir}_{2}$-Gemeinschaften ist daher aus eben dieser Zuschreibungspraxis zu erklären. Und diese erfolgt über die Logik des Systems der Personalpronomina. Die Dialektik von Exklusion und Inklusion, die für die Identitätsbildung von partikularen $\mathrm{Wir}_{2}$-Gruppen konstitutiv ist, ist also nicht an das Freund-Feind-Schema gebunden, wie dies etwa von Carl Schmitt nahegelegt wird,

68 Tugendhat 1979, S. 284.

69 Ebd., S. 71. 
der die Feindschaft der je eigenen Wir ${ }_{2}$-Gruppe zu anderen Gruppen geradezu ontologisch als ,seinsmäßige Negierung eines anderen Seins" versteht. ${ }^{70}$ Die Freund-FeindUnterscheidung ist eine nähere Bestimmung der Außenbeziehung von politischen Gemeinschaften, wobei die Unterscheidung zwischen Freund und Feind die Unterscheidung zwischen ,uns" und ,ihnen" bereits voraussetzt.

Das Wort „wir" ist kein Eigenname eines Kollektivs. Es ist kein „Nomen“, sondern ein „Pronomen“ und es wird so verwendet, daß es sich jeweils auf diejenigen bezieht, die der Sprecher damit als sein Kollektiv anspricht. Sein Gebrauch ist immer von einem Kollektiv und der Identifikation eines Sprechers mit diesem Kollektiv abhängig. Denn wenn ein anderer Sprecher aus einer anderen $\mathrm{Wir}_{2}$-Gemeinschaft auf diejenigen Bezug nimmt, die nach Ansicht des vorigen Sprechers unter dieses „wir“ fielen, dann verwendet er nicht das Wort „wir", sondern das Wort „ihr“", wenn er die Mitglieder dieser Gruppe anreden will oder das Wort „sie“, wenn er zu Dritten über die Mitglieder dieser Gruppe reden will.

Es gehört demnach konstitutiv zur Verwendung des Wortes „wir", so damit auf eine Gruppe Bezug genommen wird, die kleiner ist als die Gruppe aller vernünftigen Wesen, daß, wer „wir“" sagt, erstens weiß, daß dieselbe Gruppe von anderen Sprechern mit „,ihr“ angeredet und mit ,sie“ bezeichnet werden kann, und zweitens, daß er damit eine einzelne Gruppe von anderen Gruppen, die er mit „sie“ bezeichnet, heraushebt. Bestünde dieser Zusammenhang nicht, dann würde ,wir" keine Entität bezeichnen können. „Wir“ und „sie" würden sich nicht auf Entitäten beziehen, wenn es nicht Gruppen gäbe, die sich als solche über bestimmte Eigenschaften identifizieren ließen. In diesem Sinne gilt Quines Diktum: „no entity without identity“, keine Entität ohne Identität - wobei wir mit Tugendhat hinzufügen können: „no identity without entity“, keine Identität ohne Entität.

Nun werden jedoch Personen und Gruppen nicht unter denselben Bedingungen identifiziert, wie beobachtbare Objekte. Diese werden durch eine raum-zeitliche Identifizierung identifiziert, also numerisch, jene hingegen numerisch und qualitativ. Die Eigenart der Personen- und Gruppen- gegenüber der Objektidentifizierung erklärt sich daraus, daß Personen und Gruppen die Identitätsbedingungen und sogar die Kriterien, anhand derer sie identifiziert werden können, nicht von Haus aus erfüllen. Sie müssen ihre Identität als Person oder Gruppe erst erwerben, damit sie als eine Person oder Gruppe, und gegebenenfalls als eine bestimmte Person oder Gruppe qualitativ identifiziert werden können.

Dies ist auch der Grund, warum die qualitative Identifizierung an das normative Selbstverständnis von Personen oder Kollektiven anknüpfen muß, da ohne Rekurs auf dieses Selbstverständnis gar nicht qualitativ identifiziert werden kann. Wenn wir also Aussagen über den Gemeinsinn und das Gemeinwohl von partikularen Wir $\mathrm{W}_{2}$-Gruppen machen wollen, wenn wir etwas über das praktische Kollektivbewußtsein und damit über die Wünsche, Absichten und Überzeugungen der Personen aussagen wollen, die zu diesen Gruppen gehören, dann müssen wir uns wohl oder übel auf einen Akt der Verständigung stützen, ,wobei Alter aus der Perspektive der zweiten Person Egos expressi-

\footnotetext{
70 Schmitt 1991, S. 33.

71 Tugendhat 1979 , S. $72 \mathrm{f}$.
} 
ve Äußerung als wahrhaftig akzeptiert. Dies bedeutet, daß jemand in der kommunikativen Rolle des Sprechers mit (mindestens) einem anderen in der kommunikativen Rolle des Hörers eine interpersonale Beziehung aufnimmt, wobei sich beide im Kreise aktuell unbeteiligter, aber potentieller Teilnehmer begegnen. Die an die Perspektive der ersten, zweiten und dritten Person gebundene interpersonale Beziehung aktualisiert eine zugrundeliegende Beziehung zu einer sozialen Gruppe. “72 Während Entitäten im allgemeinen dadurch bestimmt sind, daß ein Sprecher von ihnen etwas aussagen kann, gehören $\mathrm{Wir}_{2}$-Gruppen zu der Klasse von Entitäten, die über ihre Mitglieder die Rolle eines Sprechers übernehmen und dabei in der Perspektive der ersten Person Plural den selbstbezüglichen Ausdruck ,wir" verwenden können.

\section{Konventionelle und post-konventionelle Identität}

Hermeneutische Selbstverständigungsdiskurse, die sich auf das Gemeinwohl und damit auf eine $\mathrm{Wir}_{2}$-Gemeinschaft beziehen, sind Diskurse, in denen die Beteiligten sich darüber Klarheit verschaffen müssen, wie sie sich aktuell und künftig selbst verstehen wollen - und dies schließt natürlich die Frage ein, welche von ihren Traditionen sie fortsetzen oder abbrechen wollen. Was für den Gemeinsinn im Sinne von gemeinsam geteilten Bedeutungen gilt, das gilt mutatis mutandis auch für den Gemeinsinn im Sinne der Orientierung auf das Gemeinwohl: die Seinsweise dieses gemeinsam geteilten, wiewohl prekären und gebrochenen Sinns, hat kein Sein außerhalb des Gesprächs und ist daher im Prozeß der sprachlichen Verständigung immer wieder neu herzustellen. Und dies ist auch der Grund, weshalb die Seinsweise des Gemeinsinns nur aus der performativen Perspektive derer faßbar ist, die in diesem Verständigungsprozeß über Sachund Geltungsfragen verwickelt sind.

Diese Feststellung hat für die Identitätsbedingungen von partikularen $\mathrm{Wir}_{2}$ Gemeinschaften eine wichtige Pointe. Wenn der Gemeinsinn kein Sein außerhalb dieser performativen Perspektive der Sprecher hat, dann sind die kommunitären Identitätsbedingungen von partikularen $\mathrm{Wir}_{2}$-Gemeinschaften unter den Bedingungen der Moderne post-konventionelle Identitätsbedingungen. Allein im Gespräch, also im Prozeß der sprachlichen Verständigung über die Frage, was aufs Ganze gesehen gut oder besser für die jeweilige Gruppe ist, haben der Gemeinsinn - und damit auch das Gemeinwohl und schließlich die Gemeinschaft selbst - ihr Dasein. Außerhalb der diskursiven Praxis haben sie keinen Ort: Nirgends!

Ein angemessenes Gemeinschaftskonzept muß also zunächst Raum schaffen für die „Ja“/,Nein“-Stellungnahmen bezüglich der Mitgliedschaft in partikularen Wir ${ }_{2}$ Gemeinschaften, weil das Individuum „das So-Sein und das zu ihm gehörige Gutsein“, das für die praktische Identität der Gruppe steht, in sein Selbstverständnis aufnehmen muß. Es muß also der sozialen Welt des Kollektivs selbst zugehören wollen, einer Welt, die dadurch definiert ist, daß alle Kollektivmitglieder wechselseitig voneinander fordern, in einem bestimmten Sinn von "gut“ ein gutes Mitglied der Gemeinschaft zu

\footnotetext{
${ }^{72}$ Ebd., S. 159.
} 
sein. ${ }^{73}$ Diesen Gesichtspunkt könnte man den der Mitgliedschaft nennen, da diese eben nicht ein für allemal gegeben ist, sondern immer auch aufgekündigt werden kann - im Extremfall, wo die Möglichkeit der Wahl durch externe Faktoren derart eingeschränkt ist, daß dem Individuum diese Stellungnahme verwehrt wird, durch den Freitod.

Die Angehörigkeit des Mitglieds zu partikularen $\mathrm{Wir}_{2}$-Gemeinschaften beruht auf seiner mindestens impliziten Zustimmung - im Unterschied zur Mitgliedschaft in der moralischen Gemeinschaft, die wir einem vernünftigen Wesen nicht bestreiten können, ohne ihm sein Menschsein abzusprechen. Denn dem „So-Sein und (dem) zu ihm gehörige(n) Gutsein", das das Individuum als Mitglied der Gemeinschaft in seine Identität aufnehmen muß, liegt ein Wollen zugrunde, das sich zwar nicht instrumentalistisch im Sinn einer atomistischen Ontologie verstehen läßt, das sich aber eben auch nicht traditionalistisch im Sinne einer kommunitaristischen Ontologie verstehen läßt - etwa als ein Wollen, welches sich ohne Angabe von Gründen auf die Erhaltung des Kollektivs bezieht. Das „Müssen“ bezieht sich vielmehr darauf, daß man selbst aus freien Stücken ein Mitglied einer bestimmten Gemeinschaft sein will, die durch wechselseitige Forderungen definiert ist, die sich bei allen Mitgliedern auf ein Konzept des Gutseins beziehen.

Die traditionalistische These von der normativen Vorgängigkeit der Gemeinschaft gegenüber dem Individuum muß aber nicht nur dergestalt abgeschwächt werden, daß Raum geschaffen wird für die „Ja“/,Nein“-Stellungnahmen bezüglich der Mitgliedschaft in partikularen $\mathrm{Wir}_{2}$-Gruppen. Sie muß darüber hinaus auch noch Raum schaffen für die Veränderung jener intersubjektiv geteilten Präferenzstruktur, die den Werthorizont dieser Gemeinschaft ausmacht. ${ }^{74}$ Denn eine kollektive Orientierung an Werten, die die Vorzugswürdigkeit von Gütern ausdrücken, die in bestimmten Kollektiven als erstrebenswert gelten und durch ein zielgerichtetes Handeln realisiert werden, versteht sich unter den Bedingungen eines nachidealistischen Denkens nicht mehr von selbst. Der Gemeinsinn und das Gemeinwohl sind unter den Bedingungen der Moderne zu einem Problem geworden, das sich nicht mehr mit Rekurs auf transzendente Berufungsinstanzen, sondern nur noch diskursiv lösen läßt.

Dies hat nichts mit dem seit Nietzsche beschworenen "Nihilismus" zu tun, dem Fehlen eines ,Ziel(s)“; „,es fehlt die Antwort auf das ,Warum?““”.75 Denn der „Nihilismus“, der seither als Symptom eines allgemeinen Sinnverlusts angesehen wird, also dafür, „daß die obersten Werthe sich entwerten ${ }^{46},{ }^{76}$ ist keine Krise des Sinns überhaupt wenngleich dies im Anschluß an Nietzsche von Max Weber, über Heidegger und Adorno bis hin zu Derrida immer wieder behauptet wird. Wir müssen hier genau unterscheiden, welche Werte durch diese „Nein“-Stellungnahmen entwertet werden. Und dann

\footnotetext{
73 Vgl. Tugendhat 1993, S. 60.

74 Bezüglich unserer Überzeugungen, was auf Ganze gesehen gut oder besser für uns ist, benötigen wir eine der Revision offen stehende Metaphysik, die der Veränderung unseres "Präferenzhorizonts" (von Wright 1994, S. 97 f.) in einer Weise Rechnung trägt, daß sich die mit der internen Revision unserer Überzeugungen verbundene Horizontverschiebung auch als Horizonterweiterung verstehen läßt.

75 Nietzsche 1988 , S. 350.

76 Ebd.
} 
sehen wir, daß es sich bei der Krise des Sinns, die ja auch eine Krise des Gemeinschaftssinns sein soll, ${ }^{77}$ recht besehen gar nicht um eine Krise des Sinns handelt, sondern um eine Krise des metaphysischen Sinns, der sich unter den Bedingungen der Moderne nicht mehr verbindlich machen läßt. Der Nihilismus, also die Überzeugung von der objektiven Sinn- und Wertlosigkeit des menschlichen Daseins, erklärt sich aus dem Bankrott aller objektiven Wertlehren, mit dem sich das Sinnproblem auf den Horizont der Frage zurückzieht, was für eine Person oder für ein Kollektiv aufs Ganze gesehen gut oder besser ist, eine Frage, die unabweislich an die Perspektive der ersten Person Singular oder Plural gebunden ist.

Der vermeintliche „Sinnverlust“ und damit auch der „Verfall der Werte“ wäre danach schlicht Ausdruck der Tatsache, daß die „obersten Werte“ der metaphysischen Traditionen sich entwertet haben. Entwertet haben sich diese Werte aber nicht deshalb, weil die Menschen das ,wahrhaft Wertvolle" aus den Augen verloren haben, wie dies von den Freunden der alten Werte behauptet wird, sondern deshalb, weil sich die Überzeugungen, die einst konstitutiv für den kollektiv verbindlichen und kollektiv verbindenden Wert- und Verständnishorizont einer Gemeinschaft waren, im Licht neuer Evidenzen und Gründe als unplausibel erwiesen haben - wobei von diesem Prozeß der Entwertung nie alle Werte dieser Tradition auf einmal betroffen sein können, sondern immer nur einige. Das Ende der Metaphysik ist nicht der Anfang eines nihilistischen Ödlands, in dem zwar bürgerliche Freiheiten allgemeine Verbreitung gefunden haben, in dem es aber keine kollektiv verbindenden und kollektiv verbindlichen Werte mehr gibt, sondern lediglich das Ende des Wertobjektivismus, der das Verbindliche und das Verbindende nur noch jenseits von Geschichte glaubt auffinden zu können.

Wenn dies jedoch stimmt, dann müssen wir den „Verfall der Werte“ als einen Wertewandel und diesen als eine „interne Revision von Überzeugungen ${ }^{\text {"78 }}$ verstehen, der sich auf Gründe und Überzeugungen stützt, die sich im Lichte neuer Gründe und Überzeugungen als die besseren erwiesen haben. Wenn sich nun jedoch der Wertewandel gar nicht als Verfall verstehen läßt, wenn der Werteverfall lediglich ein Ausdruck der Tatsache ist, daß sich die Überzeugungen der Menschen darüber ändern, was für sie gut ist - wobei sich Menschen hierbei natürlich auch irren können -, dann verliert die Rede vom fortschreitenden Werteverfall viel von ihrer kulturkritischen Dramatik, so daß es bezüglich des Gemeinwohls auch angemessener zu sein scheint, von einer Änderung von kollektiv geteilten Überzeugungen und Wünschen zu sprechen.

Mit der Abschwächung der traditionalistischen These von der normativen Vorgängigkeit der Gemeinschaft gegenüber den Individuen zeigt sich aber nicht nur, daß der angebliche Werteverfall als ein Wertewandel verstanden werden muß, sondern auch, daß die fehlende Antwort auf das „Ziel“" und auf das „Warum“, eine Antwort ist, die nur noch kommunikativ erzeugt werden kann. Und hierfür gibt es einen ganz einfachen Grund: Unter den Bedingungen der Moderne „,ist das gute Leben nicht mehr abtrennbar von dem, was Menschen mit freiem Willen für das gute Leben halten ${ }^{\text {". }} .{ }^{79}$ Kollektive Lebensentwürfe können daher nur noch dann vernünftig genannt werden, wenn sie

77 So Taylor 1995, S. 17.

78 Vgl. Wingert 1993, S. 164.

79 Schnädelbach 1992b, S. 218. 
erstens den tatsächlichen Lebensumständen einer Gemeinschaft entsprechen, zweitens gegenüber Kritik und neuer Erfahrung offen sind und drittens das Handeln des Kollektivs auf Situationen hin orientieren, die von den Kollektivmitgliedern aufs Ganze gesehen als gut oder besser bezeichnet werden. ${ }^{80}$

Was wir sind, ist also stets doppelt bestimmt: es ist etwas Vorgefundenes und es ist etwas Gemachtes. Es wird einerseits konstatiert und andererseits entworfen. Und genau deshalb hängt auch das, was wir sind, nicht nur davon ab, was wir glauben zu sein, sondern immer auch davon, was wir glauben sein zu können. Die Identität von partikularen $\mathrm{Wir}_{2}$-Gruppen hängt in einer Weise mit der "semantischen Autorität“ ihrer Mitglieder zusammen, daß diese auf der Basis alter Geschichten neue Geschichten über das eigene Dasein ersinnen, durch die die Bedeutung der Beziehungen zu einer Personengruppe vermindert und die Bedeutung zu einer anderen Gruppe gesteigert wird. Daher ist auch das einzige, worauf man sich von den Praktiken einer bestimmten Gemeinschaft ausgehend berufen könnte, die Praxis einer besseren Gemeinschaft.

Daher erhalten sich unter modernen Bedingungen auch nur solche Traditionen und nur solche Kollektive am Leben, an die sich die Menschen selbst binden. In einer reflexiven Moderne schließt der Bezug zur Tradition eine intentionale Variation und Selektion ein, und dies beinhaltet immer auch das Verfügen über das Tradierte, sei es im Sinn der bewahrenden oder ausschließenden Selektion. Die Freiheit in der Gemeinsamkeit kommunalen Lebens und die Freiheit ihm gegenüber - eine Freiheit zur zwanglosen Teilnahme wie zur zwanglosen Distanznahme - sind gleichursprünglich. Hier gibt es eine Wahl, die keinem Mitglied einer Gemeinschaft abgenommen werden kann - weder durch die Geschichte, noch durch das Kollektiv. In diesem Sinne ist der Mensch tatsächlich ,zur Freiheit verurteilt“" ${ }^{81}$ Wenn sich Traditionen aber nur dann erhalten können, wenn sie diskursiv artikuliert und verteidigt und ihre leitenden Werte gegenüber einer Vielzahl von Alternativen gerechtfertigt werden, dann sind unter den Bedingungen der Moderne Traditionen auf das Entgegenkommen einer dialogischen Kultur angewiesen.

Dialogische Kulturen aber sind reflexive Kulturen. Reflexivität ist ein Strukturmerkmal des Kulturellen, durch das Traditionen in den Bereich menschlicher Verfügung treten. Wenn es keine guten Gründe mehr gibt, sich an bestimmten Werten zu orientieren und Traditionen fortzusetzen, dann verlieren diese Werte ihre orientierende Funktion und diese Traditionen werden nicht fortgesetzt. Selbst die berühmte „Macht der Gewohnheit" wird daran auf Dauer nichts ändern können. Denn es gibt wohl keine Gewohnheit, die man sich nicht auch wieder abgewöhnen kann. Unter den Bedingungen der Moderne müssen auch Gewohnheiten zunehmend durch das Nadelöhr von diskursiven Revisionen hindurch. Genau dies macht die Rationalität von dialogischen Kulturen aus. Diese sind nicht deshalb rational, weil sie ein Fundament haben, das jenseits der Kritik liegt, sondern deshalb, weil es sich bei ihnen um selbstkorrigierende Unternehmungen handelt, die prinzipiell alles in Frage stellen können wenn auch nicht alles auf einmal.

\footnotetext{
${ }^{80} \mathrm{Vgl}$. Seel 1995, S. 95.

${ }^{81}$ Sartre 1985, S. 614.
} 


\section{Literaturverzeichnis}

Aristoteles (19797), Nikomachische Ethik, Aristoteles Werke Bd. 6, hg. v. H. Flashar, Berlin.

Assmann, J. (1992), Das kulturelle Gedächtnis. Schrift, Erinnerung und politische Identität in frühen Hochkulturen, München.

Benhabib, S. (1993), Demokratie und Differenz. Betrachtungen über Rationalität, Demokratie und Postmoderne, in: Gemeinschaft und Gerechtigkeit, hg. v. M. Brumlik u. H. Brunkhorst, Frankfurt/M., S. 97-116.

Brandom, R. (2000), Expressive Vernunft. Begründung, Repräsentation und diskursive Festlegung, Frankfurt/M.

Davidson, D. (1982), Rational Animals, in: Dialectica 36, S. 317-328.

Davidson, D. (1991), Subjektiv, Intersubjektiv, Objektiv, in: Merkur 512, S. 999-1014.

Gadamer, H.-G. $\left(1990^{6}\right)$, Wahrheit und Methode. Grundzüge einer philosophischen Hermeneutik, Gesammelte Werke Bd. 1, Tübingen.

Gerhardt, V. (1999), Selbstbestimmung. Das Prinzip der Individualität, Stuttgart.

Habermas, J. (1985a), Theorie des kommunikativen Handelns, 2 Bde., Frankfurt/M.

Habermas, J. (1985b), Der philosophische Diskurs der Moderne, Frankfurt/M.

Habermas, J. (1996), Die Einbeziehung des Anderen. Studien zur politischen Theorie, Frankfurt/M.

Habermas, J. (1999), Richtigkeit versus Wahrheit. Zum Sinn der Sollgeltung moralischer Urteile und Normen, in: Wahrheit und Rechtfertigung, Frankfurt/M., S. 271-318.

Heidegger, M. $\left(1979^{15}\right)$, Sein und Zeit, Tübingen.

Heidegger, M. (1981), Hölderlin und das Wesen der Dichtung, in: Erläuterungen zu Hölderlins Dichtung, Gesamtausgabe Bd. 4, hg. v. F.-W. v. Herrmann, Frankfurt/M., S. 33-48.

Hölderlin, F. (1970), Hyperion, in: Sämtliche Werke und Briefe Bd. 2, hg. v. G. Mieth, Berlin/Weimar, S. 99-268.

Kant, I. (1979) Kritik der reinen Vernunft, hg. v. R. Schmidt, Leipzig.

Kant, I. (1976), Kritik der Urteilskraft, Stuttgart.

Luhmann, N. (1986), Ökologische Kommunikation. Kann die moderne Gesellschaft sich auf ökologische Gefährdungen einstellen?, Heidelberg.

MacIntyre, A. (1994), Ist Patriotismus eine Tugend?, in: Kommunitarismus. Eine Debatte über die moralischen Grundlagen moderner Gesellschaften, hg. v. A. Honneth, Frankfurt/New York, S. 84102.

Münkler, H. (Hg., 1996), Bürgerreligion und Bürgertugend. Debatten über die vorpolitischen Grundlagen politischer Ordnung, Baden-Baden.

Nietzsche, F. (1988), Nachgelassene Fragmente 1885-1887, Kritische Studienausgabe, Bd. 12, hg. v. G. Colli u. M. Montinari, Berlin/New York.

Novalis (1978), Die Christenheit oder Europa, Schriften 3. Bd., Das philosophische Werk II, hg. v. R. Samuel, Stuttgart u. a., S. 507-524.

Plessner, H. (1955), Nachwort zu Tönnies, in: Kölner Zeitschrift für Soziologie, 7. Jg., S. 341-347.

Putnam, H. (1982), Vernunft, Wahrheit und Geschichte, Frankfurt/M.

Raulet, G. (1993), Die Modernität der ,Gemeinschaft', in: Gemeinschaft und Gerechtigkeit, hg. v. M. Brumlik u. H. Brunkhorst, Frankfurt/M., S. 72-96.

Rorty, R. (1988), Solidarität oder Objektivität? Drei philosophische Essays, Stuttgart.

Rorty, R. (1989), Kontingenz, Ironie und Solidarität, Frankfurt/M.

Rorty, R. (1994), Hoffnung statt Erkenntnis. Eine Einführung in die pragmatische Philosophie, Wien.

Rorty, R. (2001), Erwiderung auf Udo Tietz, in: Hinter den Spiegeln. Beiträge zur Philosophie von Richard Rorty, hg. v. Th. Schäfer, U. Tietz u. R. Zill, Frankfurt/M., S. 107-112.

Sandel, M. J. (1982), Liberalism and the Limits of Justice, Cambridge. 
Sandel, M. J. (1994), Die verfahrensrechtliche Republik und das ungebundene Selbst, in: Kommunitarismus. Eine Debatte über die moralischen Grundlagen moderner Gesellschaften, hg. v. A. Honneth, Frankfurt/New York, S. 18-35.

Sartre, J. P. (1985), Das Sein und das Nichts. Versuch einer phänomenologischen Ontologie, Reinbek. Scheler, M. (1954 $4^{4}$ ), Der Formalismus in der Ethik und die materiale Wertethik, Werke Bd. 2, hg. v. E. Fink u. M. Scheler, Bern.

Schmitt, C. (1991), Der Begriff des Politischen, Berlin.

Schnädelbach, H. (1992a), Über Rationalität und Begründung, in: Ders., Zur Rehabilitierung des animal rationale. Vorträge und Abhandlungen 2, Frankfurt/M., S. 61-78.

Schnädelbach, H. (1992b), Was ist Neoaristotelismus?, in: Ders., Zur Rehabilitierung des animal rationale. Vorträge und Abhandlungen 2, Frankfurt/M., S. 205-230.

Schnädelbach, H. (2001), Werte und Wertungen, in: Logos. Zeitschrift für systematische Philosophie $1-2$, S. $149-170$.

Schwemmer, O. (1986), Ethische Untersuchungen. Rückfragen zu einigen Grundbegriffen, Frankfurt/M.

Seel, M. (1995), Versuch über die Form des Glücks. Studien zur Ethik, Frankfurt/M.

Spaemann, R. (1989), Glück und Wohlwollen. Versuch über Ethik, Stuttgart.

Taylor, Ch. (1986a), Die Motive einer Verfahrensethik, in: Moralität und Sittlichkeit. Das Problem Hegels und die Diskursethik, hg. v. W. Kuhlmann, Frankfurt/M., S. 101-135.

Taylor, Ch. (1986b), Sprache und Gesellschaft, in: Kommunikatives Handeln. Beiträge zu Jürgen Habermas' ,Theorie des kommunikativen Handelns', hg. v. A. Honneth u. H. Joas, Frankfurt/M., S. $35-52$.

Taylor, Ch. (1988a), Was ist menschliches Handeln?, in: Ders., Negative Freiheit. Zur Kritik des neuzeitlichen Individualismus, Frankfurt/M., S. 9-51.

Taylor, Ch. (1994a), Quellen des Selbst. Die Entstehung der neuzeitlichen Identität, Frankfurt/M.

Taylor, Ch. (1994b), Aneinander vorbei: Die Debatte zwischen Liberalismus und Kommunitarismus, in: Kommunitarismus. Eine Debatte über die moralischen Grundlagen moderner Gesellschaften, hg. v. A. Honneth, S. 103-130.

Taylor, Ch. (1995), Das Unbehagen an der Moderne, Frankfurt/M.

Tietz, U. (1995), Sprache und Verstehen in analytischer und hermeneutischer Sicht, Berlin.

Tietz, U. (1995a), Gerechtigkeit und Solidarität, in: Die Gegenwart der Gerechtigkeit. Diskurse zwischen Recht, praktischer Philosophie und Politik, hg. v. Ch. Demmerling u. Th. Rentsch, Berlin, S. 208-231.

Tietz, U. (1996), Universalistisches Verstehen versus partikulare Lebensform. Über Identität, Moralität und die ,moralischen Quellen des Selbst', in: Philosophiegeschichte und Hermeneutik, hg. v. V. Caysa u. K. D. Eichler, Leipzig, S. 133-158.

Tietz, U. (1998), Güterethik versus Diskursethik, in: INITIAL. Zeitschrift für sozialwissenschaftlichen Diskurs 2, S. 55-70.

Tietz, U. (2001), Das principle of charity und die ethnozentristische Unterbestimmung der hermeneutischen Vernunft, in: Hinter den Spiegeln, Beiträge zur Philosophie von R. Rorty, hg. v. Th. Schäfer, U. Tietz u. R. Zill, Frankfurt/M., S. 77-106.

Tietz, U. (2002a), Verstehen und Begründen aus dem Kontext. Aspekte eines antifundamentalistischen Universalismus, Berlin.

Tietz, U. (2002b), Die Grenzen des Wir - Bausteine einer Theorie der Gemeinschaft, Frankfurt/M.

Tönnies, F. (1965), Einführung in die Soziologie, hg. v. R. Heberle, Stuttgart.

Tönnies, F. (1991), Gemeinschaft und Gesellschaft. Grundbegriffe der reinen Soziologie, Darmstadt.

Tugendhat, E. (1979), Selbstbewußtsein und Selbsterkenntnis, Frankfurt/M.

Tugendhat, E. (1993), Vorlesungen über Ethik, Frankfurt/M. 
Walzer, M. (1996), Lokale Kritik - globale Standards. Zwei Formen moralischer Auseinandersetzung, hg. v. O. Kallscheuer, Hamburg.

Weber, M. (1972a), Wirtschaft und Gesellschaft, Tübingen.

Weber, M. (1972b), Gesammelte Aufsätze zur Wissenschaftslehre, Tübingen.

Wellmer, A. (1993), Bedingungen einer demokratischen Kultur. Zur Debatte zwischen Liberalen und Kommunitaristen, in: Gemeinschaft und Gerechtigkeit, hg. v. M. Brumlik u. H. Brunkhorst, Frankfurt/M., S. 173-198.

Wellmer, A. (1995), Zur Kritik der hermeneutischen Vernunft, in: Vernunft und Lebenspraxis. Philosophische Studien zu den Bedingungen einer rationalen Kultur, hg. v. Ch. Demmerling, G. Gabriel u. Th. Rentsch, Frankfurt/M., S. 123-156.

Wingert, L. (1993), Gemeinsinn und Moral. Grundzüge einer intersubjektivistischen Moralkonzeption, Frankfurt/M.

Wolf, U. (1998), Zur Struktur der Frage nach dem guten Leben, in: Was ist ein gutes Leben? Philosophische Reflexionen, hg. v. H. Steinfath, Frankfurt/M., S. 32-46.

Wright, G. H. v. (1994) Normen, Werte und Handlungen, Frankfurt/M.

Young, I. M. (1990), Justice and the Politics of Difference, Princeton. 